\title{
Clustering and entrainment effects on the evaporation of dilute droplets in a turbulent jet
}

\author{
Federico Dalla Barba and Francesco Picano* \\ Department of Industrial Engineering, University of Padova, Via Venezia 1, 35131, Padova, Italy
}

(Received 21 July 2017; published 15 March 2018)

\begin{abstract}
The evaporation of droplets within turbulent sprays involves unsteady, multiscale, and multiphase processes which make its comprehension and modeling capabilities still limited. The present work aims to investigate the dynamics of droplet vaporization within a turbulent spatial developing jet in dilute, nonreacting conditions. We address the problem considering a turbulent jet laden with acetone droplets and using the direct numerical simulation framework based on a hybrid Eulerian-Lagrangian approach and the point droplet approximation. A detailed statistical analysis of both phases is presented. In particular, we show how crucial is the preferential sampling of the vapor phase induced by the inhomogeneous localization of the droplets through the flow. Strong droplet preferential segregation develops suddenly downstream from the inflow section both within the turbulent core and the jet mixing layer. Two distinct mechanisms have been found to drive this phenomenon: the inertial small-scale clustering in the jet core and the intermittent dynamics of droplets across the turbulent-nonturbulent interface in the mixing layer, where dry air entrainment occurs. These phenomenologies strongly affect the overall vaporization process and lead to an impressive widening of the droplet size and vaporization rate distributions in the downstream evolution of the turbulent spray.
\end{abstract}

DOI: 10.1103/PhysRevFluids.3.034304

\section{INTRODUCTION}

Turbulent sprays are complex multiphase flows involving unsteady and multiscale phenomena such as turbulence coupled with a phase transition process. The presence of two distinguished phases which mutually interact exchanging mass, momentum, and energy makes the description of the problem extremely challenging. If combustion is considered, chemical reactions and heat release add some clear complexities. In this scenario, a satisfactory comprehension of the turbulent spray dynamics has not yet been achieved, and existing models' capabilities for applications are still limited $[1,2]$. Nevertheless, the research progress in this field is crucial for several industrial applications as well as from an environmental point of view. A typical example can be found in the development of high-efficiency and low-emission internal combustion engines. In these applications liquid fuel is directly injected into the combustion chamber where the vaporization of fuel droplets occurs together with chemical reactions within the turbulent gaseous environment. The formation of pollutants in turbulent spray combustion is related to complex multiscale phenomena that involve fluctuations of temperature and reactants concentrations. In particular, soot formation occurs through a pyrolysis process in fuel-rich regions that experience high temperature without enough oxidizer to react [3-5]. This can be observed within droplet clusters, where the concentration of the fuel droplets can be even a thousand times higher than its bulk value leading to a peak in the fuel vapor concentration. Hence, in order to predict and model the soot formation process, an improved understanding of the

\footnotetext{
*Corresponding author: francesco.picano@unipd.it
} 
mechanisms that govern the distribution of droplets and fuel-oxidizer mixture within turbulent jet sprays is mandatory.

A phenomenological description of the overall evolution of the spray dynamics can be found in the review of Jenny et al. [1]. The process starts with the primary atomization of a high-velocity liquid jet. As the liquid flow is ejected from a duct into a gaseous environment, interface instabilities such as Kelvin-Helmholtz and Rayleigh-Taylor fragmentize the jet into large drops and liquid ligaments [6]. In their downstream evolution liquid ligaments and drops are subjected to aerodynamic forces induced by the velocity difference at the liquid-gas interface. The stresses induced by aerodynamic forces produce a further breakup of the liquid phase (secondary atomization) giving origin to a system of small droplets dispersed within the gaseous turbulent phase. The atomization process occurs in a so-called dense regime and terminates when the surface tension prevails on aerodynamic stresses preventing further fragmentation. At this step a dilute regime establishes: droplet mutual interactions (e.g., collisions and coalescence) are negligible, but the effect of droplets on the carrier flow is still considerable $[7,8]$. While in the dense regime the vaporization rate is negligible, in dilute conditions the vaporization process becomes significant. In this phase of the turbulent spray evolution, most of the liquid evaporates. Moreover, small droplets evolve preserving a spherical shape due to the dominance of the surface tension on the aerodynamic stresses. Even if the presence of droplets exerts a significant effect on the flow in terms of mass, momentum, and energy balance, at this step the droplet size is usually below or comparable to the smallest scales of the turbulent flow so the point droplet approximation applies [9]. Hence, in dilute conditions the mathematical description of droplet-laden flows lends itself particularly well to a hybrid Eulerian-Lagrangian description. The Navier-Stokes equations govern the dynamics of the continuous phase if distributed sink-source terms are considered in order to represent the mass, momentum, and energy exchanges between the Eulerian carrier phase and Lagrangian point droplets.

One of the most peculiar phenomenologies characterizing dispersed multiphase turbulent flows in dilute conditions is the preferential segregation of particles and droplets as a result of the interaction of their inertia with the carrier phase turbulent dynamics (see, e.g., Toschi and Bodenschatz [10]). The mechanisms at the origin of the preferential segregation in free flows have been widely investigated in the literature for both solid particles and liquid droplets. The intensity of the small-scale segregation of solid particles in homogeneous turbulence is found to be driven by the Stokes number $\mathrm{St}_{\eta}=\tau_{p} / \tau_{\eta}$, with $\tau_{p}$ the particle relaxation time and $\tau_{\eta}$ the Kolmogorov time scale. More specifically, the intensity of the small-scale clustering is maximum when the particle relaxation time is of the order of the

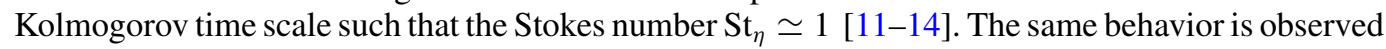
for evaporating droplets which behave as inertial particles [15]. Concerning turbulent jets, a mean accumulation of the dispersed phase has been observed at specific distances from the inflow section both experimentally [16] and numerically [17]. Even if the preferential concentration of a dispersed phase has been well characterized in homogeneous turbulence, a satisfactory comprehension of the effect of this phenomenology on the overall vaporization process within turbulent jet sprays has not yet been achieved and constitutes the main focus of this paper.

From a theoretical and numerical point of view, one of the first descriptions of the vaporization of spherical droplets dragged by a gaseous phase flow was addressed in the seminal works of Spalding [18] and Godsave [19]. Fixing the environmental vapor concentration, they found that the droplet surface decreases linearly with time (the d-square law). Maxey and Riley [20] derived an equation for the motion of a finite size spherical particle or droplet at low Reynolds number. The equation accounts for the Stokes drag, added-mass effect, and buoyancy force. Dealing with a dispersed phase with a density much higher than that of the fluid, the Stokes relaxation time $\tau_{p}$ is an appropriate time scale in order to describe the drag force effect on the dispersed phase dynamics [21,22]. Abramzon and Sirignano [23] proposed an improved model for droplet vaporization, considering nonuniform and time-dependent environmental conditions, taking into account forced convection, molecular diffusion, and the Stefan flow contribution to the vapor transport from the droplet surface to neighboring environment. Even if several models can be found in literature for the simulation of turbulent evaporating sprays in the Reynolds averaged Navier-Stokes (RANS) or large-eddy 
simulation (LES) frameworks $[1,24]$, these models lack in capabilities to accurately reproduce complex phenomena such as strong polydispersity and small-scale clustering of droplets [2]. Despite the highly demanding computational resources, the use of direct numerical simulation (DNS) allows one to capture the whole physics of the spray vaporization process in order to understand the complex phenomena involved. In this context, Mashayek [25] adopted an Eulerian-Lagrangian approach in order to perform a DNS of low Mach number, homogeneous shear turbulent flows laden with droplets. Miller and Bellan [26] reported a DNS of a confined three-dimensional, temporally developing gas mixing layer laden with evaporating hydrocarbon droplets at subsonic Mach number. Reveillon and coworkers [15] studied the effect of preferential droplet accumulation on the evaporation in isotropic turbulence showing that different regimes take place depending on the droplet concentration. Recently Bukhvostova et al. [27] considered the DNS of a turbulent channel flow of a mixture of air and water vapor laden with water droplets. The work focuses on the comparison between the performances of an incompressible and a low Mach number asymptotic formulation in reproducing the flow dynamics. Even if the two formulations show a good qualitative agreement, the low Mach number formulation is found to be crucial in order to obtain a reliable quantitative prediction of heat and mass transfer.

The prototypal flow for an evaporating spray is constituted by a turbulent free jet which is characterized by the effect of environmental gas entrainment. In more detail, a turbulent jet is constituted by a rotational turbulent core which is continuously entrained by the surrounding irrotational fluid [28]. In sprays, the entrained dry flow dilutes the vapor concentration and controls the vaporization process. This phenomenology was found to be of critical importance also in natural phenomena. One example consists in the effect of entrainment in stratocumulus clouds where it constitutes a driving parameter in the determination of cloud lifetimes and in turn even in regulating planetary-scale properties [29]. Moreover, the fast grow rate of the droplet size spectrum in turbulent warm clouds is a challenging, still not understood problem in cloud physics [30,31], despite its importance in determining extreme weather conditions.

To the best of the authors' knowledge, a fundamental study of the effects of the entrainment in an evaporating turbulent jet spray in combination with an analysis of the preferential segregation effect is still missing. This work aims to cover this lack considering DNS data of an evaporating turbulent spray, using a two-way coupling approach between the two phases and accounting for the entrainment effect. The numerical algorithm adopts a hybrid Eulerian-Lagrangian approach and the point droplet approximation. In addition, the effect of density variation is accounted by a low Mach number formulation of the Navier-Stokes equations, even though at lower Reynolds number and in a slightly different flow configuration, thermodynamic and droplet parameters have been selected to be similar to the experiments of Refs. [32,33]. Strong droplet preferential segregation is observed over the whole downstream evolution of the spray, which induces a preferential sampling of the vapor concentrated regions. Two different mechanisms are found to drive this process, the former due to the inertial clustering, the latter related to the dynamics of the jet entrainment. This last mechanism is found to be crucial in the outer part of the jet core, where the evaporation peaks, and strongly impacts the vaporization process which is characterized by an extreme widening of the droplet size spectrum.

\section{NUMERICAL METHOD AND MODEL}

In the present paper we report the results of the DNS of a turbulent evaporating spray in a hybrid Eulerian-Lagrangian framework. The point droplet approximation is adopted in the two-way coupling conditions. The governing equations for the Eulerian gaseous phase consist in a low Mach number asymptotic formulation of the Navier-Stokes equations in an open environment. By the use of this approach arbitrary density variations can be accounted neglecting acoustics [34]. Within this framework, the Navier-Stokes equations adopted in the present study can be written as

$$
\frac{\partial \rho}{\partial t}+\nabla \cdot(\rho \mathbf{u})=S_{m},
$$




$$
\begin{gathered}
\frac{\partial}{\partial t}\left(\rho Y_{v}\right)+\nabla \cdot\left(\rho Y_{v} \mathbf{u}\right)=\nabla \cdot\left(\rho \mathcal{D} \nabla Y_{v}\right)+S_{m}, \\
\frac{\partial}{\partial t}(\rho \mathbf{u})+\nabla \cdot(\rho \mathbf{u} \otimes \mathbf{u})=\nabla \cdot \boldsymbol{\tau}-\nabla P+\mathbf{S}_{p}, \\
\nabla \cdot \mathbf{u}=\frac{\gamma-1}{\gamma} \frac{1}{p_{0}}\left[\nabla \cdot(k \nabla T)+S_{e}-L_{v}^{0} S_{m}\right], \\
p_{0}=\rho R_{m} T,
\end{gathered}
$$

where $\mathbf{u}, \rho, T, p_{0}$, and $P$ are the velocity, total density, temperature, thermodynamic pressure, and hydrodynamic pressure fields of the carrier vapor-gas mixture, respectively, and $Y_{v}=\rho_{v} / \rho$ is the vapor mass fraction field, with $\rho_{v}$ the vapor partial density. The thermal conductivity of the carrier mixture and the binary mass diffusion coefficient of the vapor into the gas are $k$ and $\mathcal{D}$, respectively. The Eulerian carrier phase is assumed to be governed by the state equation (5) where $R_{m}=\bar{R} / W_{m}$ is the gas constant of the mixture with $W_{m}$ its molar mass and $\bar{R}$ the universal gas constant. $\gamma=$ $c_{p} / c_{v}$ is the specific heat ratio of the carrier mixture with $c_{p}$ and $c_{v}$ the specific heat capacity at constant pressure and volume, respectively. The viscous stress tensor is $\tau=\mu\left(\nabla \mathbf{u}+\nabla \mathbf{u}^{T}\right)-$ $(2 / 3) \mu(\boldsymbol{\nabla} \cdot \mathbf{u}) \mathbf{I}$, with $\mu$ the dynamic viscosity. The hypothesis of calorically perfect chemical species is assumed, and a reference temperature $T_{0}=0 \mathrm{~K}$ is fixed in order to estimate the enthalpies. Under this assumption we denote the latent heat of vaporization of the liquid phase evaluated at the reference temperature $T_{0}=0 \mathrm{~K}$ as $L_{v}^{0}$. Consistently with previous studies in this field [25-27], the effect of the dispersed phase on the gaseous carrier phase is accounted for by three sink-source coupling terms in the right-hand side of the mass, momentum, and energy equations, $S_{m}, \mathbf{S}_{p}$, and $S_{e}$, respectively:

$$
\begin{gathered}
S_{m}=-\sum_{i=1} \frac{d m_{d, i}}{d t} \delta\left(\mathbf{x}-\mathbf{x}_{d, i}\right), \\
\mathbf{S}_{p}=-\sum_{i=1} \frac{d}{d t}\left(m_{d, i} \mathbf{u}_{d, i}\right) \delta\left(\mathbf{x}-\mathbf{x}_{d, i}\right), \\
S_{e}=-\sum_{i=1} \frac{d}{d t}\left(m_{d, i} c_{l} T_{d, i}\right) \delta\left(\mathbf{x}-\mathbf{x}_{d, i}\right),
\end{gathered}
$$

where $\mathbf{x}_{d, i}, m_{d, i}, \mathbf{u}_{d, i}$, and $T_{d, i}$ are the $i$ th droplet position, mass, velocity, and temperature, respectively, and $c_{l}$ is the liquid specific heat. The sum is taken over the whole droplet population located within the domain, and the delta function expresses that the sink-source terms act only at the domain locations occupied by the point droplets. In the numerical algorithm, the Eulerian terms (6)-(8) are calculated in correspondence of each grid node by volume averaging the mass, momentum, and energy sources from all droplets located within the cell volume centered around the considered grid point.

The droplets are treated as rigid evaporating spheres, and the liquid phase properties (e.g., temperature) are assumed to be uniform inside each droplet. In addition, being the present work restricted to the investigation of dilute conditions, droplet mutual interactions (e.g., collisions, coalescence) are neglected. Given their importance, the validity of the preceding assumptions will be briefly discussed here. Under these hypotheses, the dynamics of the droplets is completely described by the following Lagrangian equations:

$$
\begin{gathered}
\frac{d \mathbf{x}_{d}}{d t}=\mathbf{u}_{d}, \\
\frac{d \mathbf{u}_{d}}{d t}=\frac{f}{\tau_{d}}\left(\mathbf{u}-\mathbf{u}_{d}\right), \quad f=1+0.15 \operatorname{Re}_{\mathrm{d}}^{0.687}
\end{gathered}
$$




$$
\begin{gathered}
\frac{d m_{d}}{d t}=-\frac{1}{3} \frac{m_{d}}{\tau_{d}} \frac{\mathrm{Sh}}{\mathrm{Sc}} H_{m}, \quad H_{m}=\ln \left(1+B_{m}\right), \\
\frac{d T_{d}}{d t}=\frac{1}{3 \tau_{d}}\left[\frac{\mathrm{Nu}}{\operatorname{Pr}} \frac{c_{p, g}}{c_{l}}\left(T-T_{d}\right)-\frac{\mathrm{Sh}}{\mathrm{Sc}} \frac{L_{v}}{c_{l}} \mathrm{H}_{m}\right],
\end{gathered}
$$

where $c_{p, g}$ is the gas specific heat capacity at constant pressure and $\tau_{d}=2 \rho_{l} r_{d}^{2} /(9 \mu)$ is the droplet relaxation time, with $\rho_{l}$ the liquid density. $L_{v}$ is the latent heat of vaporization evaluated at the droplet temperature. The Schiller-Naumann correlation is adopted in Eq. (10) in order to account for the effect of the finite droplet Reynolds number on the drag. The mass diffusivity and the thermal conductivity are accounted for in Eqs. (11) and (12) through the Schmidt and Prandtl numbers, respectively: $\mathrm{Sc}=\mu /(\rho \mathcal{D})$ and $\operatorname{Pr}=\mu /\left(c_{p} k\right)$. The Nusselt number, $\mathrm{Nu}_{0}$, and the Sherwood number, $\mathrm{Sh}_{0}$, are estimated as a function of the droplet Reynolds number, $\operatorname{Re}_{\mathrm{d}}=2 \rho\left\|\mathbf{u}-\mathbf{u}_{d}\right\| r_{d} / \mu$, according to the Frössling correlation:

$$
\begin{aligned}
& \mathrm{Nu}_{0}=2+0.552 \operatorname{Re}_{\mathrm{d}^{\frac{1}{2}}} \operatorname{Pr}^{\frac{1}{3}}, \\
& \mathrm{Sh}_{0}=2+0.552 \operatorname{Re}_{\mathrm{d}^{\frac{1}{2}} \operatorname{Pr}^{\frac{1}{3}} .}
\end{aligned}
$$

A correction is then applied to $\mathrm{Nu}_{0}$ and $\mathrm{Sh}_{0}$ in order to account for the Stefan flow [23]:

$$
\begin{gathered}
\mathrm{Nu}=2+\frac{\left(\mathrm{Nu}_{0}-2\right)}{F_{T}}, \quad F_{T}=\frac{\left(1+B_{t}\right)^{0.7}}{B_{t}} H_{t}, \\
\mathrm{Sh}=2+\frac{\left(\mathrm{Sh}_{0}-2\right)}{F_{M}}, \quad F_{M}=\frac{\left(1+B_{m}\right)^{0.7}}{B_{m}} H_{m} .
\end{gathered}
$$

The parameters $H_{m}$ and $H_{t}$ are defined as $H_{m}=\ln \left(1+B_{m}\right)$ and $H_{t}=\ln \left(1+B_{t}\right)$ with $B_{m}$ and $B_{t}$ the Spalding mass and heat transfer number, respectively, the former being the driven parameter for the vaporization rate:

$$
\begin{gathered}
B_{m}=\frac{\left(Y_{v, s}-Y_{v}\right)}{\left(1-Y_{v, s}\right)}, \\
B_{t}=\frac{c_{p, v}}{L_{v}}\left(T-T_{d}\right),
\end{gathered}
$$

where $c_{p, v}$ is the constant pressure coefficient of the vapor, $Y_{v}$ is the vapor mass fraction field evaluated at droplet center, and $Y_{v, s}$ is the vapor mass fraction evaluated at droplet surface. This latter parameter corresponds to the mass fraction of the vapor in a saturated vapor-gas mixture at the droplet temperature. In order to estimate $Y_{v, s}$ we assume the Clausius-Clapeyron relation to apply:

$$
\chi_{v, s}=\frac{p_{\text {ref }}}{p_{0}} \exp \left[\frac{L_{v}}{R_{v}}\left(\frac{1}{T_{\text {ref }}}-\frac{1}{T_{d}}\right)\right],
$$

with $\chi_{v, s}$ the vapor molar fraction at droplet surface, $p_{0}$ the thermodynamic pressure, $p_{\text {ref }}$, and $T_{\text {ref }}$ an arbitrary reference pressure and temperature and $R_{v}=\bar{R} / W_{l}$ the vapor gas constant. The saturated vapor mass fraction is then

$$
Y_{v, s}=\frac{\chi_{v, s}}{\chi_{v, s}+\left(1-\chi_{v, s}\right) \frac{W_{g}}{W_{l}}},
$$

where $W_{g}$ and $W_{l}$ are the molar mass of the gas and liquid phases, respectively.

In the present flow configuration, the ex post analysis of the cumulative distribution function of the droplet Reynolds number computed over the whole droplet population shows that $\mathrm{Re}_{\mathrm{d}}$ varies in the range $0<\operatorname{Re}_{\mathrm{d}}<2$ with a probability $P \geqslant 0.99$ (not reported). In these conditions the Nusselt 
TABLE I. Thermodynamic and physical properties of acetone and dry air. The pressure $p_{0}$ is constant both over time and in space due to the low Mach number formulation and open environmental conditions. The temperature, $T_{0}$, the bulk velocity, $U_{0}$, and dynamic viscosity, $\mu$, are evaluated at the inflow section. The parameters $c_{p, g}, c_{p, v}$, and $c_{l}$ are the specific heat capacity at constant pressure of the gas and vapor and the liquid specific heat capacity, respectively. $W_{g}, W_{l}, k_{g}$, and $k_{l}$ are the molar mass and the thermal conductivities of the gas and the liquid. $\mathcal{D}$ is the mass binary diffusion coefficient of the vapor into the gas. $\rho_{l}$ and $L_{v}$ are the liquid density and latent heat of vaporization evaluated at the inflow temperature, respectively.

\begin{tabular}{lccccc}
\hline \hline$p_{0}$ & {$[\mathrm{~Pa}]$} & 101300 & $W_{g}$ & {$[\mathrm{~kg} / \mathrm{mol}]$} & $2.90 \times 10^{-2}$ \\
$T_{0}$ & {$[\mathrm{~K}]$} & 275.15 & $W_{l}$ & {$[\mathrm{~kg} / \mathrm{mol}]$} & $5.81 \times 10^{-2}$ \\
$U_{0}$ & {$[\mathrm{~m} / \mathrm{s}]$} & 8.10 & $k_{g}$ & {$[\mathrm{~W} /(\mathrm{m} \mathrm{K})]$} & $2.43 \times 10^{-2}$ \\
$\mu$ & {$[\mathrm{kg} /(\mathrm{m} \mathrm{s})]$} & $1.75 \times 10^{-5}$ & $k_{l}$ & {$[\mathrm{~W} /(\mathrm{m} \mathrm{K})]$} & $1.83 \times 10^{-1}$ \\
$c_{p, g}$ & {$[\mathrm{~J} /(\mathrm{kg} \mathrm{K})]$} & 1038 & $\mathcal{D}$ & {$\left[\mathrm{m}^{2} / \mathrm{s}\right]$} & $1.10 \times 10^{-5}$ \\
$c_{p, v}$ & {$[\mathrm{~J} /(\mathrm{kg} \mathrm{K})]$} & 1300 & $\rho_{l}$ & {$\left[\mathrm{~kg} / \mathrm{m}^{3}\right]$} & 800 \\
$c_{l}$ & {$[\mathrm{~J} /(\mathrm{kg} \mathrm{K})]$} & 2150 & $L_{v}$ & {$[\mathrm{~J} / \mathrm{kg}]$} & 530000 \\
\hline \hline
\end{tabular}

and Sherwood numbers, regulating the convective heat and mass transfer rates, slightly vary around $\mathrm{Nu}=2$ and $\mathrm{Sh}=2$. Then, according to Bukhvostova et al. [27], the droplet Biot number, which quantifies the ratio of heat convection and heat conduction, can be estimated as $\mathrm{Bi}_{\mathrm{d}} \simeq k_{g} / k_{l} \simeq 0.13$, with $k_{g}$ and $k_{l}$ the thermal conductivities of gas and liquid, respectively. Hence, the temperature in each droplet can be assumed constant. The bulk volume fraction of the liquid phase prescribed at the inflow section is $\Phi=8 \times 10^{-5}$. However, inside droplet clusters the liquid volume fraction may be significantly greater than its bulk value so that the effect of the droplet collisions and coalescence could be relevant. This aspect has been found to be of critical importance in turbulent channel flows where, besides small-scale clustering, turbophoresis increases the mean liquid volume fraction at the wall even by orders of magnitude. In these flow configurations, even with bulk volume fractions of the order of $10^{-4}$, the effect of droplet collisions is significant (see, e.g., Kuerten and Vreman [35]). In the present work collisions and coalescences have not been considered and will be investigated in dedicated future studies. Nonetheless, the ex post analysis of the local liquid phase volume fraction shows that the $99 \%$ of the overall events are located within the interval $0<\Phi<2 \times 10^{-4}$. Hence, in the large part of the present flow configuration the volume fraction can be assumed to be small enough such that the effect of the droplet collisions and coalescence is negligible.

The present DNS reproduces the dynamics of liquid acetone droplets dispersed within a turbulent air-acetone vapor jet. The gas-vapor mixture is injected into an open environment through an orifice of radius $R=5 \times 10^{-3} \mathrm{~m}$ at a bulk velocity $U_{0}=8.1 \mathrm{~m} / \mathrm{s}$. Liquid acetone monodisperse droplets with an initial radius $r_{d, 0}=6 \mu \mathrm{m}$ are randomly distributed over the inflow section. The ambient pressure is set to $p_{0}=101300 \mathrm{~Pa}$ while the injection temperature is fixed to $T_{0}=275.15 \mathrm{~K}$ for both the droplets and the carrier mixture. The injection flow rate of the gaseous phase is kept constant fixing a bulk Reynolds number $\operatorname{Re}=2 U_{0} R / v=6000$, with $v=1.35 \times 10^{-5} \mathrm{~m}^{2} / \mathrm{s}$ the kinematic viscosity. At the inflow section a nearly saturated condition is prescribed for the air-acetone vapor mixture, $S=Y_{v} / Y_{v, s}=0.99$, with $Y_{v}$ the actual vapor mass fraction and $Y_{v, s}\left(p_{0}, T_{0}\right)$ the vapor mass fraction saturation level evaluated at the actual inflow temperature and thermodynamic pressure. The acetone mass flow rate is set by the mass flow rate ratio $\Psi=\dot{m}_{\text {act }} / \dot{m}_{\text {air }}=0.28$, with $\dot{m}_{\text {act }}=\dot{m}_{\text {act }, l}+\dot{m}_{\text {act }, v}$ the sum of liquid and gaseous acetone mass flow rates and $\dot{m}_{\text {air }}$ the gaseous one. The correspondent bulk volume fraction of the liquid phase is set to $\Phi=8 \times 10^{-5}$, as previously mentioned. All the thermodynamic and physical properties of the vapor, gas, and liquid phases are reported in Table I. The thermodynamic conditions of the flow at the inlet section are comparable to that adopted in the well-controlled experiments on dilute coaxial sprays published by the group of Masri and coworkers (see, e.g., Chen et al. [32]). They used acetone droplets dispersed in air at the temperature of $275.15 \mathrm{~K}$ in both nonreactive and reactive conditions, but at higher Reynolds number. 
TABLE II. Taylor $\lambda_{c}$ and Kolmogorov $\eta_{c}$ microscales along the jet axis at different $z / R$.

\begin{tabular}{cccc}
\hline \hline & $z / R=20$ & $z / R=40$ & $z / R=60$ \\
\hline$\lambda_{c} / R$ & 0.21 & 0.37 & 0.49 \\
$\eta_{c} / R$ & 0.013 & 0.021 & 0.03 \\
\hline \hline
\end{tabular}

The numerical code consists of two different modules. An Eulerian algorithm directly evolves the gaseous phase dynamics solving the Low-Mach number formulation of the Navier-Stokes equations (1)-(5) (see, e.g., Refs. [36,37] and references therein for validations and tests). A second-order central finite differences scheme is adopted on the staggered grid for space discretization [38], while the temporal evolution is performed by a low-storage third-order Runge-Kutta scheme. A Lagrangian solver evolves the droplet mass, momentum, and temperature laws (9)-(12). Actually, in order to mitigate numerical stability issues when the droplet size becomes small, the Lagrangian solver numerically evolves the following equation for the droplet square radius instead of the equivalent equation (11):

$$
\frac{d r_{d}^{2}}{d t}=-\frac{\mathrm{Sh}}{\mathrm{Sc}} \frac{\rho_{g}}{\rho_{l}} v_{g} H_{m} .
$$

The temporal integration uses the same Runge-Kutta scheme adopted by the Eulerian algorithm. Second-order accurate polynomial interpolations are used to calculate the Eulerian quantities at the droplet positions. The computational domain consists of a cylinder extending for $2 \pi \times 22 R \times 70 R$ in the azimuthal, $\theta$, radial, $r$ and axial, $z$, directions. The domain has been discretized by $N_{\theta} \times$ $N_{r} \times N_{z}=128 \times 225 \times 640$ points using a nonequispaced, staggered mesh in the radial and axial direction. The mesh has been stretched in order to be of the order of the Kolmogorov length scale $\eta=\left(v^{3} / \epsilon\right)^{1 / 4}(\epsilon$ turbulent energy dissipation) in the downstream evolution of the spray. At the jet inlet section the maximum of the typical mesh size on the Kolmogorov length, $\Delta / \eta=\left[\left(r \Delta_{\theta}\right) \Delta_{r} \Delta_{z}\right]^{1 / 3} / \eta$, is about 2.4 and is kept around this value during the downstream jet evolution. It should be noted that the initial resolution is critical for turbulent jets since in the far field all length scales increase linearly with the axial distance, while the Reynolds number is constant. Even in the present case, we found that the Taylor-scale Reynolds number is approximatively constant, i.e., $\operatorname{Re}_{\lambda}=u^{\prime} \lambda / v \simeq 77 \pm 14$ $\left(\lambda=\sqrt{(15 v / \epsilon)} u^{\prime}\right.$ and $u^{\prime}$ the root-mean-square of velocity fluctuations), while the typical turbulent scales increase almost linearly; see Table II. The flow is injected at the center of one base of the cylindrical domain and streams out towards the other base. A convective condition is adopted at the outlet, and an adiabatic traction-free condition is prescribed at the side boundary. This side boundary condition makes the entrainment of external fluid possible, which is dry air in the present case. Time-dependent inflow boundary conditions are prescribed. A fully turbulent velocity is assigned at the jet inflow section (Dirichlet condition) by means of a cross-sectional slice of a companion fully developed DNS of a pipe flow. The flow is injected through a center orifice while the remaining part of the domain base is impermeable and adiabatic. The turbulent periodic pipe extends for $2 \pi \times 1 R \times 8 R$ in the azimuthal, $\theta$, radial, $r$, and axial, $z$, directions. The domain is discretized with an equispaced staggered mesh containing $N_{\theta} \times N_{r} \times N_{z}=128 \times 80 \times 128$ nodes in order to match the corresponding jet computational grid at the pipe discharge. A sketch of the cylindrical domain together with the turbulent periodic pipe is provided in Fig. 1. A statistical characterization of the inflow is provided at the end of this section along with additional data for validation; see Fig. 2.

The DNS reported in the present paper was performed on Galileo cluster at CINECA Institute, Bologna, Italy. Galileo is a tier-1 cluster IBM NeXtScale made of 516 nodes of dual eight-cores Intel Haswell processors. To perform the simulation 32 cores distributed over two nodes were used for a total amount of about 10000 core hours, corresponding to a bulk time of about one month. The time step is set to $\Delta t / t_{0}=0.002$ where the reference time scale is $t_{0}=R / U_{0}=6.2 \times 10^{-4} \mathrm{~s}$. In order to achieve the prescribed mass flow rate, 69 acetone monodisperse droplets with radius 


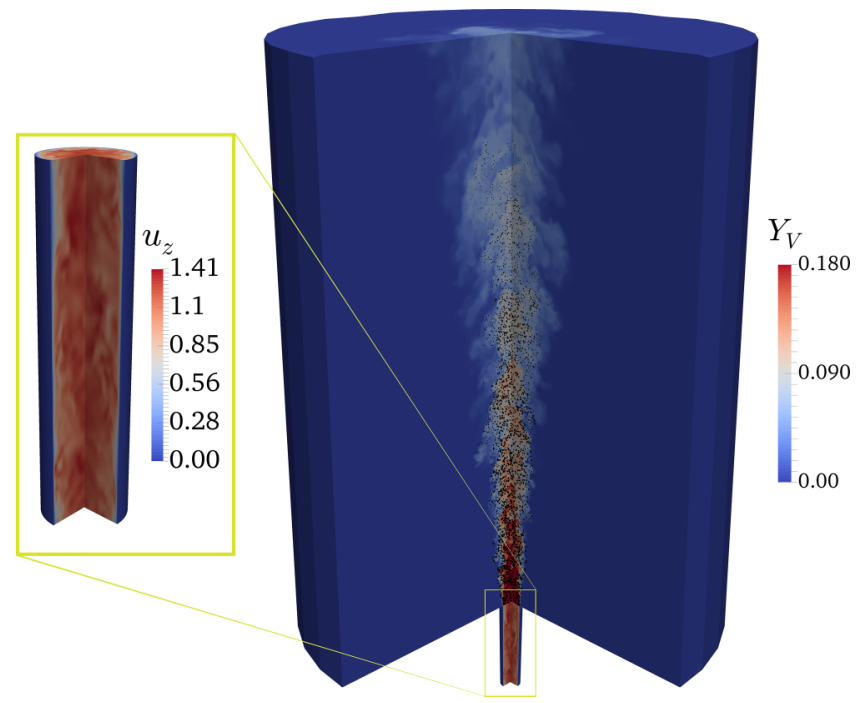

FIG. 1. A sketch of the 3D cylindrical domain and the turbulent periodic pipe. A representative ensemble of the whole droplet population is plotted with black points. The colors contour the vapor mass fraction field within the jet and the axial instantaneous velocity of the turbulent pipe, respectively.

$r_{d, 0}=6 \mu \mathrm{m}$ are randomly distributed over the inflow section at each step of the time integration algorithm. The injection velocity of each droplet is prescribed to be equal to the local velocity of the turbulent carrier phase. The simulation is initialized considering only the single-phase flow until statistical steady conditions have been attained (about $500 R / U_{0}$ time scales). From this step, droplets are continuously injected, and the simulation is run for about $200 R / U_{0}$ time scales in order to reach

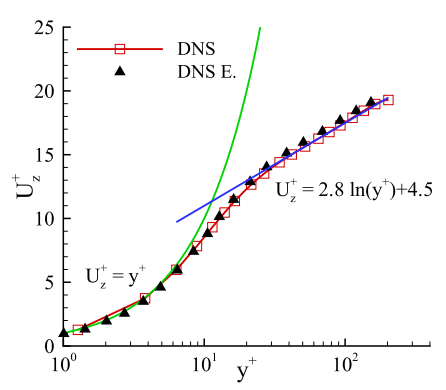

(a)

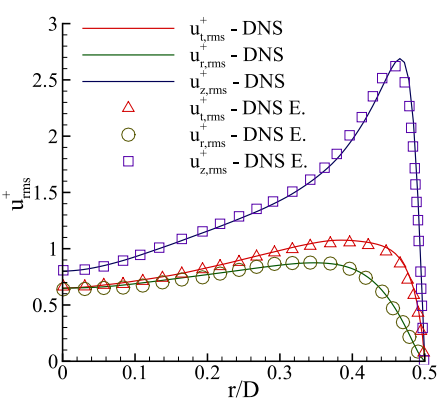

(b)

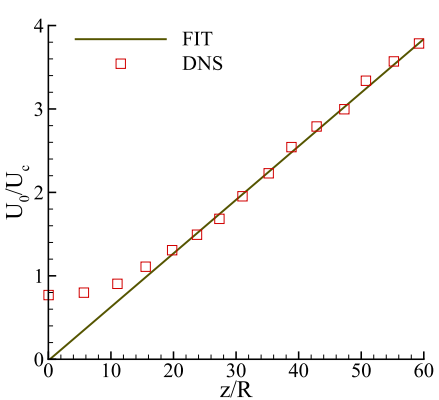

(c)

FIG. 2. (a) Mean axial velocity profile of the turbulent pipe expressed in internal coordinates. $U^{+}=U_{z} / u_{\tau}$ and $y^{+}=y u_{\tau} / v$ with $u_{\tau}=\sqrt{\tau_{w} / \rho}$ the friction velocity, $U_{z}=\left\langle u_{z}\right\rangle$ the mean axial velocity, and $y=R-r$ the distance to the wall. The plot is log-linear. Within the viscous sublayer the velocity profile follows the linear law $U^{+}=y^{+}$. In the outer layer the $\log$ law $U^{+}=1 / k \ln (y+)+B$ provides a good fit of the mean velocity profile with $k=2.8$ and $B=4.5$. The red square-dotted line represents the present DNS data, and the black triangles correspond to the DNS reported in Eggels et al. [39]. (b) Root mean square of the azimuthal $u_{t, \mathrm{rms}}^{+}$, radial $u_{r, \mathrm{rms}}^{+}$, and axial $u_{z, \mathrm{rms}}^{+}$velocities of the turbulent pipe expressed in internal coordinates vs $r / D$. The numerical data set is compared to the fully turbulent pipe DNS of Eggels et al. [39] reported by symbols. (c) Inverse of the mean centerline axial velocity of the unladen jet at $\operatorname{Re}=3000, U_{0} / U_{c}$, with $U_{0}$ the jet bulk velocity at the inflow section. In the far field of the jet, $z / R>20$, the centerline axial velocity follows a linear trend, $U_{0} / U_{c} \simeq 1 /(2 B)\left(z / R-z_{0} / R\right)$. The decaying constant estimated from the interpolation of the data set in the far field is $B \simeq 7.5$ while the virtual origin of the jet is found to be $z_{0} / R \simeq 0.25$. 
a statistical steady condition for the two-phase evaporating flow before collecting the data set. All the statistics presented in the following are computed considering around 100 samples separated in time by $R / U_{0}=1$.

In the following, some numerical issues arise concerning the numerical stability of the evolution of tiny droplets. Droplets whose radius decreases under a fixed stability threshold, $r_{d, t h}$, are discarded from the simulation. In the present case, the threshold radius is set to $r_{d, t h}=0.5 \mu \mathrm{m}$ with the time step set to $\Delta t / t_{0}=0.002$. With the initial droplet radius set to $r_{d, 0}=6 \mu \mathrm{m}$, this means that droplets are suppressed only after the $99.95 \%$ of their initial mass has evaporated. In the preliminary small-scale tests we found that changing this threshold from $r_{d, t h}=0.5 \mu \mathrm{m}$ to $r_{d, t h}=0.75 \mu \mathrm{m}$ does not affect the results in similar conditions. Within the nonresolved droplet framework, which is adopted in the present simulation, the droplet diameter must be smaller than the Kolmogorov length scale and the mesh size. Since the droplet diameter decreases in the downstream evolution of the spray, the most critical zone for this condition to be satisfied is the inlet region in proximity to the axis. In this region the ratio between the droplet diameter and the mesh characteristic size is $d_{p} / \Delta \simeq 0.33$, with $\Delta=\left[\left(r \Delta_{\theta}\right) \Delta_{r} \Delta_{z}\right]^{1 / 3}$.

In order to prove the reliability of the simulation we report some statistics concerning both the turbulent periodic pipe DNS, which provides the jet inflow condition, and the corresponding single-phase jet DNS. It should be noted that the resolution of the turbulent pipe flow simulation matches that of the initial part of the jet. Figures 2(a) and 2(b) provide a sketch of the mean axial velocity and the root-mean-square azimuthal, radial, and axial velocities within the turbulent periodic pipe expressed in internal coordinates. The friction Reynolds number obtained through the estimation of the actual wall friction in the turbulent pipe is $\operatorname{R} e_{\tau}=u_{\tau} R / v=203$, with $u_{\tau}$ the friction velocity, $v$ the kinematic viscosity, and $R$ the pipe radius. The actual Reynolds number based on the bulk velocity is set to $\operatorname{Re}=U_{0} R / v=3000$, consistently with the jet bulk Reynolds number. A best fit of the logarithmic velocity distribution of the pipe flow is given by $U^{+} \simeq 2.8 \ln \left(y^{+}\right)+4.5$. Results are in close agreement with both the numerical and experimental findings of Eggels et al. [39] in similar conditions. Figure 2(c) provides the inverse of the normalized mean centerline axial velocity of the jet, $U_{0} / U_{c}$. In the far field of a turbulent jet the average centerline velocity is known to be inversely proportional to the axial distance from the inlet:

$$
\frac{U_{0}}{U_{c}}=\frac{1}{2 B}\left(\frac{z}{R}-\frac{z_{0}}{R}\right)
$$

where $B$ is an empirical, nonuniversal, constant and $z_{0}$ is the so-called virtual origin of the jet, representing the location of an equivalent point source of momentum originating the jet in the far field. We estimate the values of the decaying constant, $B$, and the position of the virtual origin of the jet, $z_{0}$, by interpolating the mean centerline velocity of the jet in the far field, $z / R>20$; see the figure caption. Fitting values are similar with those found for round jets, especially those generated by a fully turbulent inflow [16,40]). The capabilities of the numerical code concerning the reproduction of the evaporation dynamics have been preliminarily tested considering two different test cases. The former case concerns a liquid water droplet carried by a laminar dry air jet. In these extremely dilute conditions the Spalding d-square law is a valid analytical solution for the time evolution of the droplet radius; see Fig. 3(a). In the latter case, a water droplet freely falling in wet air is considered, and the numerical solution for the evolution of the temperature is compared to an experimental data set; see Fig. 3(b).

\section{RESULTS AND DISCUSSIONS}

A general overview of the instantaneous vapor mass fraction field together with the instantaneous distribution of the droplet population is provided in Fig. 4. Droplets populate only the turbulent jet core, while they are not present in the outer dry region. In addition, the droplet distribution is strongly inhomogeneous over the whole downstream evolution of the spray and clustering is apparent. There are several mechanisms driving the preferential concentration of droplets in a turbulent flow, the 


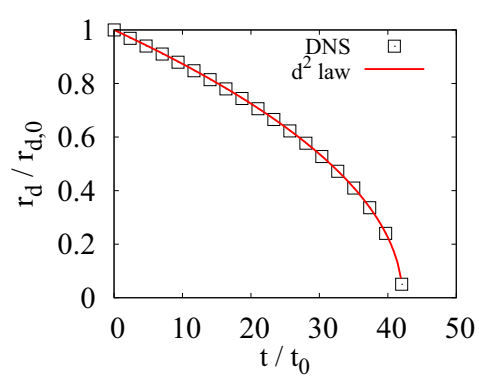

(a)

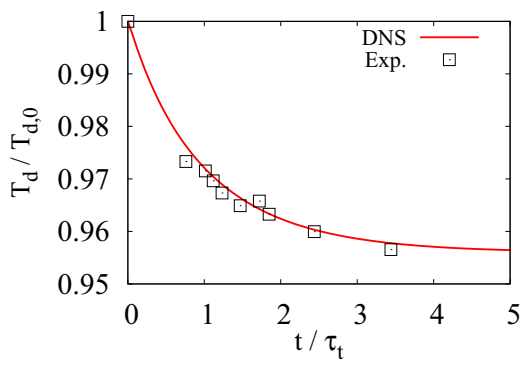

(b)

FIG. 3. (a) Evolution over time of the radius of a single water droplet in a dry air laminar jet. The ambient pressure and temperature are set to $p=101300 \mathrm{~Pa}$ and $T=273.15 \mathrm{~K}$. The inlet radius and the bulk velocity of the jet are, respectively, $R=5 \times 10^{-3} \mathrm{~m}$ and $U_{0}=1.7 \mathrm{~m} / \mathrm{s}$. The droplet is injected at the local carrier phase velocity. The initial droplet radius is set to $r_{d, 0}=5 \mu \mathrm{m}$ and the temperature to $T_{d, 0}=273.15 \mathrm{~K}$. The droplet radius and the time are scaled by the droplet initial radius and the reference time scale $t_{0}=R / U_{0}$, respectively. The continuous line represents the analytical solution computed by the Spalding d-square law, $r_{d} / r_{d, 0}=\sqrt{1-k t / t_{0}}$, where $k=\left(\rho \operatorname{Sh} R^{2}\right) /\left(\operatorname{Re} \rho_{l} \operatorname{Sc} r_{d, 0}^{2}\right) \ln \left(1+B_{m}\right)$. The symbols represent the numerical results. (b) Temperature evolution over time of a water droplet freely falling in air at pressure $p=101300 \mathrm{~Pa}$, temperature $T=301.45 \mathrm{~K}$, and relative humidity $\chi=0.22$. Droplet is initially at rest with an initial temperature, $T_{d, 0}$, equal to the environmental air one. The temperature is scaled by the initial droplet temperature, $T_{d, 0}$, while time is scaled by droplet thermal relaxation time, $\tau_{t}$. This latter is defined as the time required by droplet temperature to change by the $63 \%$ of its total change between initial temperature and regime temperature. The continuous line represents the result of the simulation while the symbols report an experimental data set [41]. The regime temperature of droplet corresponds to the ventilated wet bulb temperature at prescribed environmental pressure and actual temperature, which is $T_{w b}=288.15 \mathrm{~K}$.

most relevant of them being the small-scale clustering [10,42], the accumulation of droplets along jet axis [16,17], and, as we will show below, the intermittent droplet dynamics in the jet mixing layer. The small-scale clustering consists in a phase segregation occurring when the dissipative time scale of the flow is of the same order of the droplet inertial time scale. In addition, within turbulent jets, a mean accumulation of the dispersed phase has been observed at specific distances from the inflow section. This location is determined by the matching of the droplet time scale and the local large-scale time of the jet, which quadratically decreases with the downstream axial distance [16,17]. Independently by the mechanisms driving the droplet segregation, the vapor mass fraction increases rapidly inside the clusters of evaporating droplets due to their high local concentration. As the vapor concentration increases, the local evaporation rate is reduced. The vaporization process may even be completely blocked if the vapor concentration reaches the saturation level, $Y_{v, s}\left(p_{0}, T\right)$. In this case a nonevaporating, fully saturated core appears around the cluster [43]. It is then clear how the preferential segregation phenomenology can strongly affect the overall vaporization process (e.g., evaporation length) by locally reducing the rate of vaporization.

Figure 5(a) provides the average distribution of the liquid mass fraction over the spray, $\Psi=$ $m_{l} / m_{g}$, where $m_{l}$ and $m_{g}$ are the mean mass of the liquid acetone and of the gaseous phase inside each mesh cell. The overall vaporization length can be defined as the axial distance from the inflow section where the $99 \%$ of the injected liquid mass has transitioned to the vapor phase. According to this definition, the vaporization process is terminated at about $z / R \simeq 44$. The volume fraction $\Phi$, also reported in the same plot, shows small and streamwise diminishing values, so droplet-droplet interactions are expected to be globally negligible, as assumed. However, the liquid mass fraction is significant in the spray core affecting the turbulent gaseous phase in this region. This distribution is consistent with the field of the average saturation field, $S$, reported in Fig. 5(b). The flow is almost saturated near the inlet section due to the prescribed inflow conditions, then the saturation level gently decreases in the downstream evolution, maintaining a sharp gradient towards the outer jet 


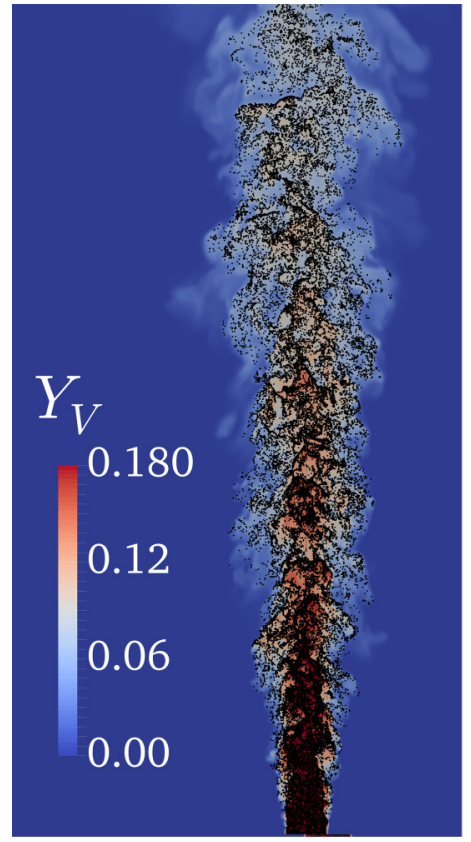

(a)
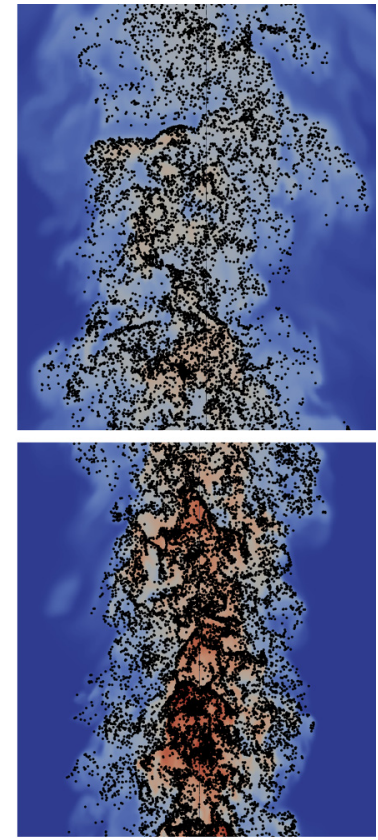

(b)

FIG. 4. (a) A radial-axial slice of the turbulent spray. The black points represent a subset of the whole droplet population constituted by droplets located within a distance $h / R=0.01$ from the slice plane. Each point size is proportional to the corresponding droplet radius (scale factor 100). The carrier phase is contoured according to the instantaneous vapor mass fraction field, $Y_{v}$, which is bounded between 0 and 0.18 , the former corresponding to the dry condition and the latter to the $99 \%$ saturation level prescribed at inlet. (b) Enlargements of two different jet regions centered at $z / R=20$ (lower panel) and $z / R=30$ (upper panel).

region. Indeed, the turbulent spray is constituted by a spreading and slowly decaying turbulent core which is surrounded by the dry and irrotational environmental gas. The turbulent core is continuously entrained by the environmental air, which mixes with the turbulent air-vapor mixture, thus reducing the vapor concentration. Since the inner core fluid cannot reach the outer region, the spray core shows a higher saturation level over the whole downstream evolution of the flow. The effect of the dry air entrainment is crucial on the overall vaporization process. The dilution of the vapor concentration is indeed fundamental in order to allow the vaporization process to advance. The mean droplet radius and vaporization rate distributions are reported in Figs. 6(a) and 6(b), respectively. According to the discussed entrainment effect, the droplet vaporization rate is maximum in the mixing layer separating the jet core and outer regions. The peak value is reached in the shear layer immediately downstream the inflow section, where large droplets enter in direct contact with the dry environmental air. Consequently, at each axial distance from the jet inlet, the largest droplets can be found in the spray core, where the vaporization process is slowed by the high vapor concentration, while the smallest ones can be found towards the outer jet region where the vaporization proceeds faster.

The power required by the vaporization process is proportional to the evaporation rate. Indeed, the transition of the liquid acetone to vapor requires an amount of energy per unit mass equal to the latent heat of vaporization. The overall energy required by the vaporization process is provided by the internal energy of both the gaseous carrier phase and the liquid dispersed phase, thus resulting in an overall cooling of the spray in the downstream evolution. The average distribution of the carrier phase and the droplet temperature is reported in Fig. 7. In the outer spray region the small droplets, surrounded by low-saturated gas, are colder than the core droplets due to the higher evaporation 


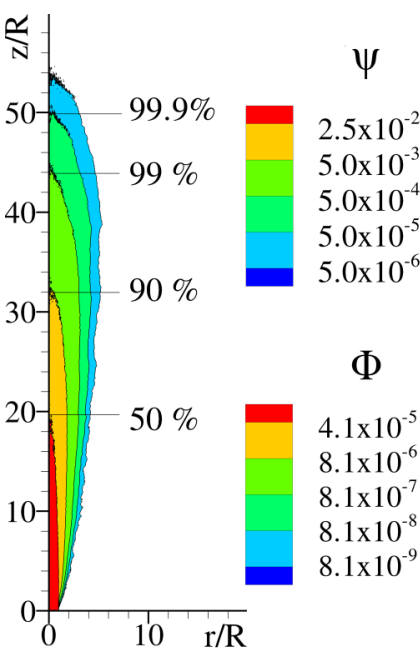

(a)

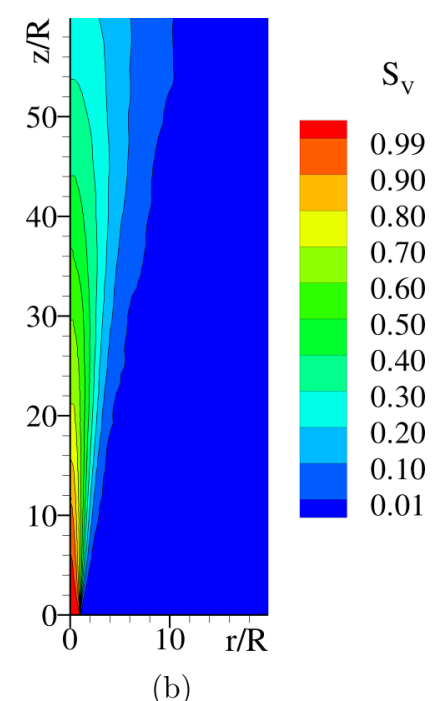

(b)

FIG. 5. (a) Mean liquid mass fraction, $\Psi=m_{l} / m_{g}$, where $m_{l}$ and $m_{g}$ are the mean mass of liquid acetone and air inside each mesh cell, respectively. It should be noted that each isoline of the mass fraction field is also an isoline of the liquid volume fraction field, $\Phi=\Psi \rho_{g} / \rho_{l}$, with $\rho_{l} / \rho_{g}$ the mean density ratio. The labels show different distances from the jet inlet section, $z / R$, in correspondence of which the $50 \%, 90 \%, 99 \%$, and 99.9\% of the injected liquid mass is evaporated. (b) Average saturation field, $S=Y_{v} / Y_{v, s}$, where $Y_{v}$ is the actual vapor mass fraction field and $Y_{v, s}=Y_{v, s}\left(p_{0}, T\right)$ is the value of vapor mass fraction corresponding to the local saturation condition.

rate. Nevertheless, the carrier phase shows an opposite behavior: the spray core is sensibly colder than the shear layer, and the minimum gas temperature can be observed between $z / R=30$ and $z / R=40$. This behavior is due to the distribution of the liquid phase mass fraction. Figure 8 reports the mean distributions of the mean mass source term, $S_{m}$, and the mean sensible energy source term, $S_{e, s}=S_{e}-L_{v}^{0} S_{m}$. Near the jet inlet, the mass and energy exchanges peak in the mixing layer. The vaporization rate is indeed extremely small in the spray core where a nearly saturated condition subsists. Nevertheless, the maxima of the energy and mass exchange distributions are located in the spray core, where the liquid mass represents a significant part of the overall spray mass. Hence, the cooling effect due to vaporization is much more intense in this region where a large amount of droplets slowly evaporates. We have noted the existence of a strong preferential segregation of the dispersed phase, focusing in particular on the effect of this inhomogeneous distribution on the overall vaporization process dynamics. In the literature, several approaches have been proposed in order to measure the intensity of the droplet segregation (see, e.g., Shaw et al. [44]). We measure the intensity of the clustering in each point of the inhomogeneous turbulent jet spray using the following clustering index [45]:

$$
K=\frac{\overline{(\delta n)^{2}}}{\bar{n}}-1
$$

where $\bar{n}$ and $\overline{(\delta n)^{2}}$ are the mean and the variance of the number of droplets in a small sampling volume $\Delta V$, respectively. In order to compute this statistic a Cartesian, equispaced sampling mesh has been considered. The cells are cubic with an edge size of $L / R=0.2$. If droplets were completely randomly located, their distribution would be determined by a Poisson process in which mean and variance coincide. Hence, where clustering is not present and particles are randomly distributed, $K=0$. On the other hand, if $K>0$ the variance exceeds the mean value, indicating that droplets preferentially segregate in clusters. Figure 9 (a) shows the clustering index, $K$, computed over the 


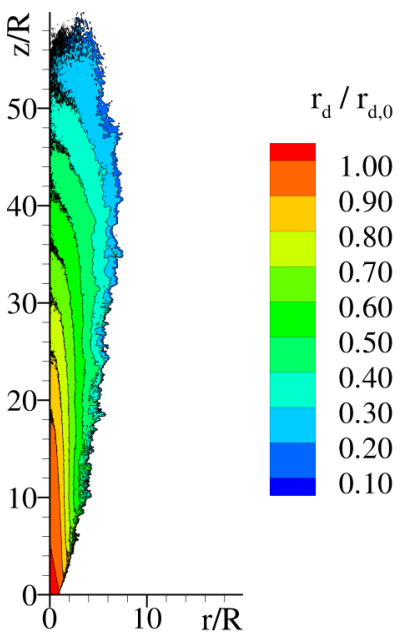

(a)

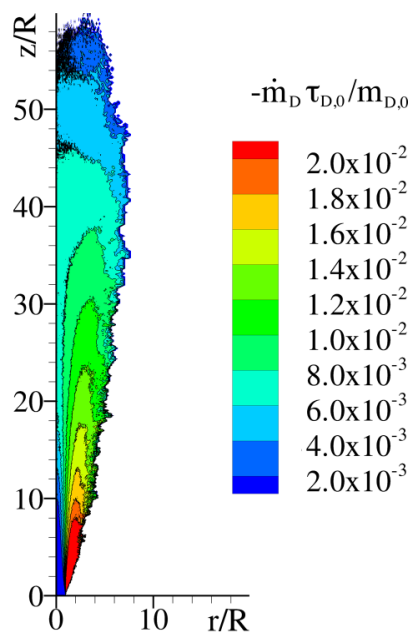

(b)

FIG. 6. (a) Mean droplet radius rescaled by the droplet initial radius $r_{d, 0}=6 \mu \mathrm{m}$. (b) Mean droplet vaporization rate divided by the reference scale defined as $\dot{m}_{d, 0}=m_{d, 0} / \tau_{d, 0}$ with $m_{d, 0}$ the initial droplet mass and $\tau_{d, 0}$ the initial droplet relaxation time.

whole spray domain. Large positive values of $K$ correspond to a strong preferential segregation of droplets. It should be noted that droplets are initially randomly located and develop clustering in their downstream evolution. In particular, near the inflow section $K$ assumes positive values only in the mixing layer. In this region the local droplet concentration is intermittent because of the fluctuation of the turbulent-nonturbulent interface which separates the turbulent core, populated by droplets, and the outer region depleted of droplets. The air regions entrained from the environment in the jet core are almost droplet-free and enhance the fluctuation levels of the droplet concentration even in the

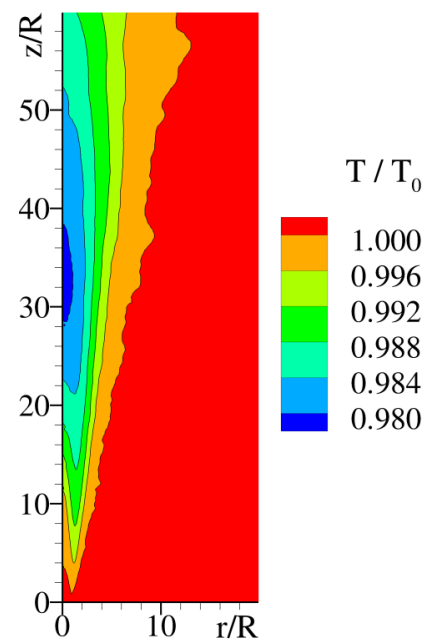

(a)

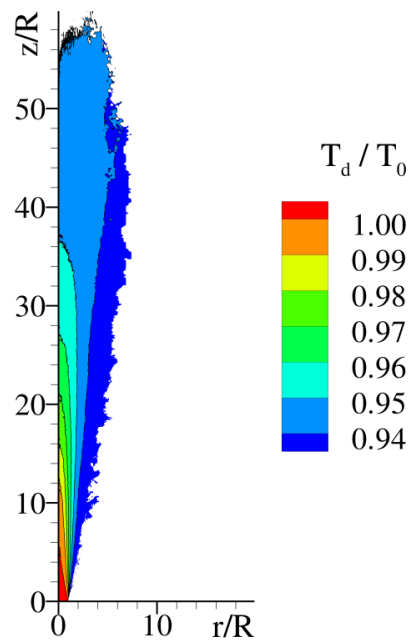

(b)

FIG. 7. (a) Mean gas phase temperature, $T$, rescaled by the injection temperature, $T_{0}$. (b) Mean droplet temperature, $T_{d}$, rescaled by droplet initial temperature, $T_{d, 0}$. The reference temperature scales are equal, $T_{d}=T_{d, 0}$. 


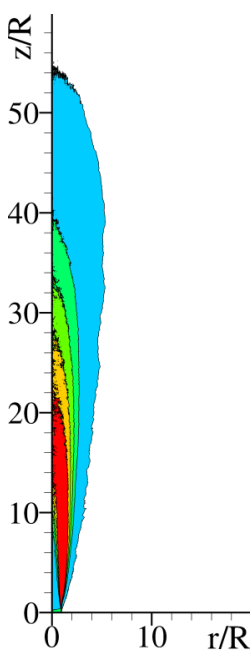

(a)

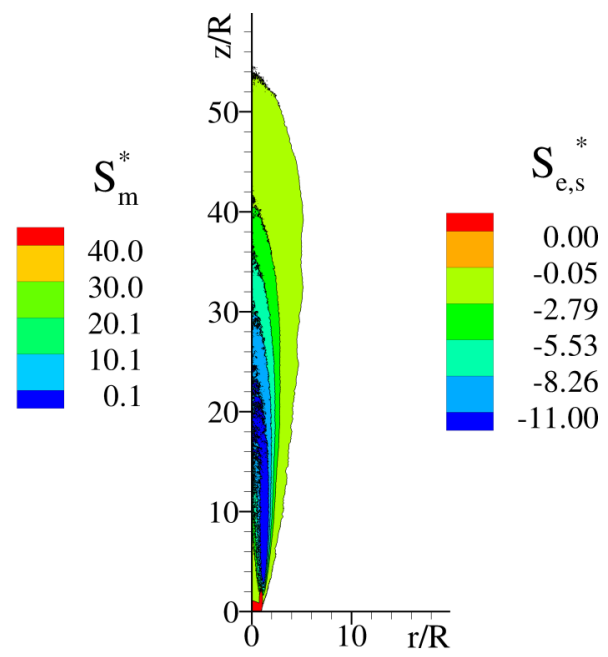

(b)

FIG. 8. (a) Mean nondimensional mass source term, $S_{m}^{*}=S_{m} R^{3} \tau_{d, 0} / m_{d, 0}$, with $R^{3}$ the inlet radius, and $m_{d, 0}$ and $\tau_{d, 0}$ the initial mass and relaxation time of the monodisperse droplets, respectively. (b) Mean nondimensional sensible energy source term, $S_{e, s}^{*}=\left(S_{e}-L_{v}^{0} S_{m}\right) R^{3} \tau_{d, 0} /\left(m_{d, 0} c_{l} T_{d, 0}\right)$, with $L_{v}^{0}$ the latent heat of vaporization, $T_{d, 0}$ the initial droplet temperature, and $c_{l}$ the liquid-specific heat capacity.

jet core (see the snapshots reported in Fig. 4). Further downstream clustering appears in the whole turbulent jet core. We attribute this phenomenology both to the developing of small-scale turbulent clustering and to the intermittent droplet dynamics in the mixing layer.

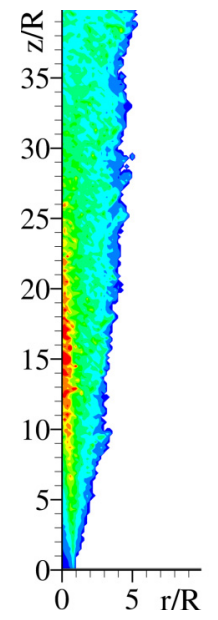

(a)

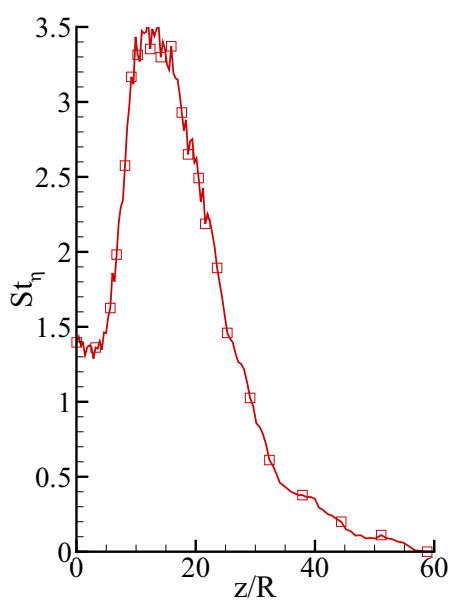

(b)

FIG. 9. (a) Droplet clustering index, $K$. (b) Evolution over the jet axis of the mean droplet Stokes number based on the Kolmogorov dissipative scale, $\mathrm{St}_{\eta}=\tau_{d} / \tau_{\eta}$. The turbulent dissipation is computed in correspondence of each mesh node in the range $0<r / R<0.2$. The local dissipative time scale is then adopted in order to estimate the Stokes number of droplets located within the correspondent cell. The average of these values is then considered as the mean droplet Stokes number on the jet axis at a given distance from the inflow section, $z / R$. 
The main mechanism driving the small-scale clustering relies on the competition between the droplet inertia and the Stokes drag. The drag tends to trail the droplets according to the highly convoluted local turbulent structures while the droplet finite inertia prevents them to follow exactly the turbulent flow motion. By this mechanism droplets heavier than the fluid tend to be ejected from the vortex cores [12]. The small-scale droplet distribution is governed by the Stokes number, $\mathrm{St}_{\eta}$, which is defined as the ratio of droplet response time, $\tau_{d}$, and the characteristic time of the dissipative scale, $\tau_{\eta}=(\nu / \epsilon)^{1 / 2}$. The droplets with $\mathrm{St}_{\eta} \gg 1$ act as ballistic particles with respect to the smallest scales of turbulence. These droplets move across the smallest turbulent structures being only weakly perturbed and showing a negligible small-scale preferential segregation. On the other hand, droplets with $\mathrm{St}_{\eta} \ll 1$ act as passive tracers which move according to the local turbulent motion without exhibiting clustering. Preferential segregation is maximum when $\mathrm{St}_{\eta} \sim 1$. Figure 9(b) provides the Stokes number, $\mathrm{St}_{\eta}$, of the droplets located within a small radial distance from the jet centerline, i.e., $r / R=0.2$. The Stokes number decreases in the downstream evolution of the spray assuming unity value around $z / R \simeq 25$. Hence, we infer that preferential segregation is promoted by the intermittent entrainment process until $z / R \simeq 25$. Further downstream, the small-scale clustering adds its contribution to the overall droplet segregation dynamics. In order to quantify the importance of the droplet preferential segregation on the evaporation process we provide a comparison between the mean vapor concentration field felt by the droplets (droplet conditioned), $Y_{V, D C}$, and the unconditional Eulerian one, $Y_{V, U} . Y_{V, D C}$ is obtained through the ensemble average of the vapor mass fraction field conditioned by the droplet presence in a given point. Figure 10 reports the radial profiles of $Y_{V, D C}$ and $Y_{V, U}$ at different axial distances from the jet inflow, $z / R$. Globally, the vapor mass fraction sampled by the droplets is often higher than the correspondent unconditional value. We will show that the preferential sampling operated by the droplets on the vapor concentration field can be related to different mechanisms. The primary contribution is given by the vapor self-produced by the droplets. Indeed, given the small Stokes number, the vapor atmosphere surrounding each droplet moves together with it increasing the vapor concentration sampled by the droplet itself. The small-scale clustering and the intermittent jet entrainment process add a significant contribution to this phenomenon that will be discussed in the following.

When droplets travel in clusters, even a small rate of evaporation suddenly brings to saturation the vapor cloud surrounding the cluster itself. This phenomenon occurs in all turbulent evaporating flows as already observed by Reveillon and Demoulin [43]. In the first part of the jet, i.e., $z / R<20$, we note that the unconditional mean vapor concentration curve and the droplet conditional one are almost identical in the turbulent jet core while they differ in the outer jet layer. This behavior can be understood considering that clustering is weak near the inflow with the exception of the mixing layer. Further downstream, we note that the droplet conditioned vapor concentration is, in average, higher of about $10 \%-60 \%$ than the unconditioned one even in the jet core. We remark that in this region the turbulent small-scale clustering is strong. To better characterize the droplet dynamics in the mixing layer, we need to discern between the inner turbulent jet core and the irrotational outer region. The two regions are separated by an almost sharp fluctuating layer, the so-called turbulentnonturbulent interface [28], that is highly convoluted over a wide range of turbulent scales. The most used observable adopted to characterize the two regions is the local enstrophy, $\zeta^{2}=\|\nabla \times \vec{u}\|^{2}$. The inner turbulent core is characterized by large fluctuations of enstrophy, while in the outer region enstrophy is null. Thus, fixing an enstrophy threshold, $\zeta_{t h}$, it is possible to distinguish if a point is located into the turbulent region or not:

$$
I(\vec{x}, t)=H\left[\zeta^{2}(\vec{x}, t)-\zeta_{t h}^{2}\right]
$$

with $H$ the Heaviside function. $I=1$ denotes a turbulent event, while $I=0$ an irrotational one. It has been shown [28] that the interface separating the rotational and irrotational regions is almost sharp and the conditional statistics weakly depend on the threshold value. Consistently, we observed a negligible influence on the statistics changing the value of $\zeta_{t h}^{2}=0.36 U_{0}^{2} / R^{2}$ by a factor 2 (this threshold value is around one order of magnitude smaller than the typical enstrophy values in the range $20<z / R<30$ ). Adopting the index $I$ we can define an enstrophy conditional average for 


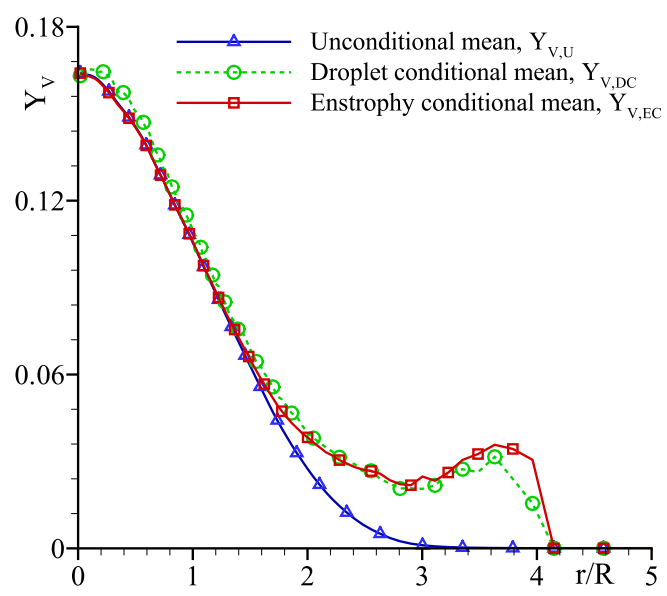

(a)

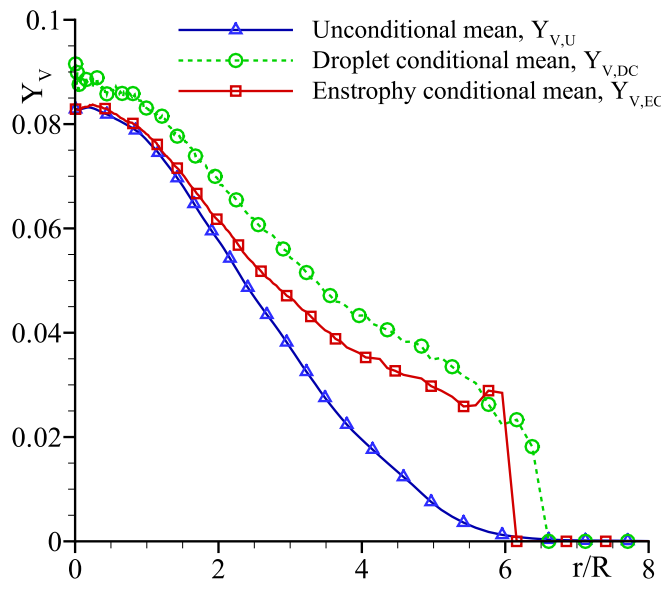

(c)

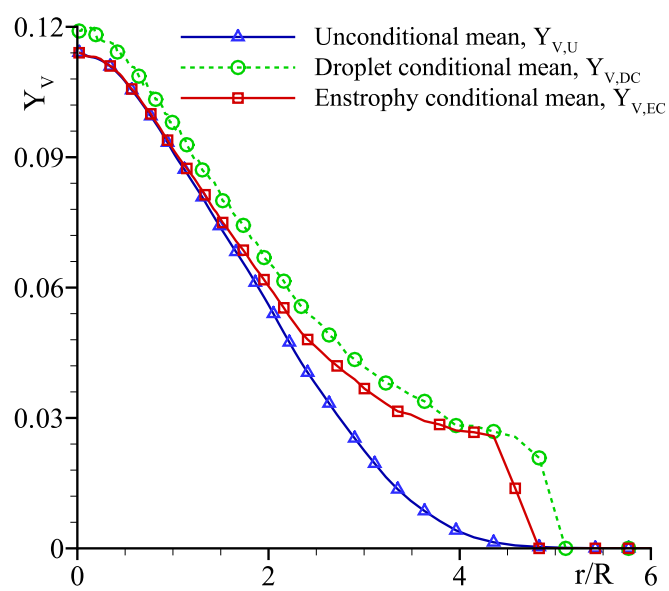

(b)

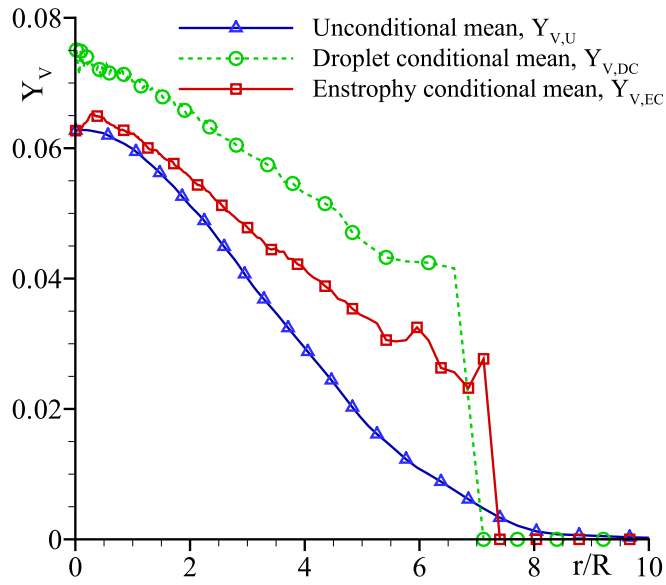

(d)

FIG. 10. Radial profiles of the average vapor mass fraction field at four different axial distances from the origin: (a) $z / R=10$, (b) $z / R=20$, (c) $z / R=30$, and (d) $z / R=40$. Each plot shows the enstrophy-threshold conditional average, $Y_{V, E C}$, the droplet-presence conditional average, $Y_{V, D C}$, and the unconditional Eulerian one, $Y_{v}$. The enstrophy-threshold conditional average is calculated by sampling the vapor mass fraction only over turbulent core events $(I=1)$, that is, when local enstrophy exceeds a fixed threshold. $Y_{V, D C}$ is the vapor concentration field obtained by a conditional average on the droplet presence in a given point.

the vapor concentration field, $Y_{V, E C}$, by sampling the vapor mass fraction only over turbulent core events. Besides the unconditioned and the droplet conditioned statistics, Fig. 10 also provides $Y_{V, E C}$, which can be seen as the mean concentration field of the turbulent region, called in the following turbulent conditioned statistics and droplet conditioned statistics, respectively. In the first part of the jet, i.e., $z / R \leqslant 20$, we note that the unconditional and both the conditional statistics are almost identical in the jet core. Hence, the vapor self-produced by the droplets weakly affects the dynamics in this region (the jet inflow is nearly saturated). However, in the shear layer, the turbulent conditioned mean vapor profile is similar to the droplet conditioned curve and is higher than the unconditional one. This indicates that droplets traveling towards the outer jet region are surrounded by a gaseous envelopment rich with vapor ejected from the turbulent jet core. Simultaneously, droplet-free dry air regions are engulfed in the jet core enhancing the fluctuation of droplet and vapor concentrations. Hence, on average, droplets evaporating in the mixing layer do not feel the unconditional mean vapor 


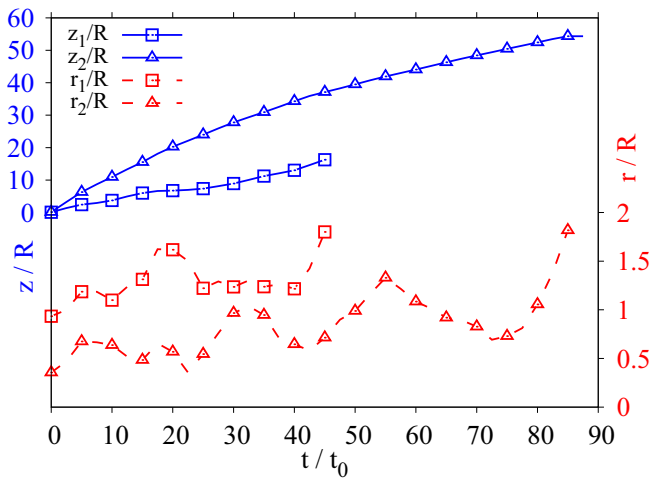

(a)

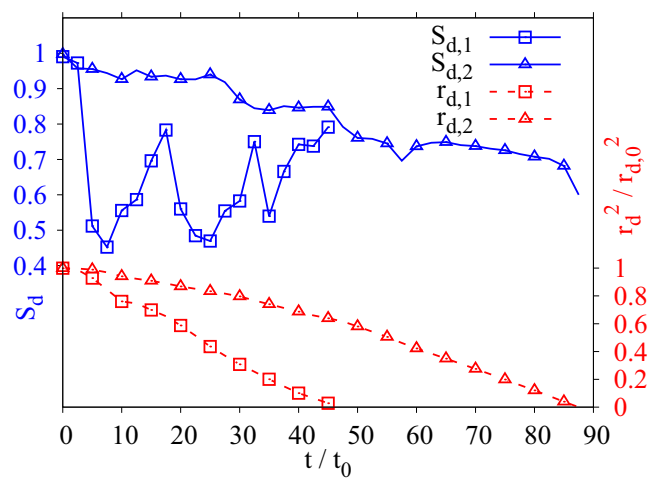

(b)

FIG. 11. (a) Radial and axial positions of two droplets " 1 " and " 2 ": the former has completely evaporated around $t / t_{0} \simeq 45$, the latter at $t / t_{0} \simeq 85$. (b) Square droplet radius, $r_{d}^{2} / r_{d, 0}^{2}$, and saturation, $S_{d}$, of the two droplets 1 and 2 .

concentration, but a higher level. The contribution of this preferential sampling mechanism appears dominant in the mixing layer, while the effect of the small-scale clustering appears more evident in the far field evolution of the jet core, i.e., $z / R>20$. In fact, as shown in Fig. 10(c) and 10(d), the droplet conditional mean vapor concentration profiles reveal the same shape of the turbulent averages, but translated of a nearly constant value. The combination of the two phenomenologies strongly reduces the vaporization rate with respect to that estimated using unconditioned statistics. We believe that, both these dynamics need to be accounted in the modeling of jet spray evaporation in order to improve the accuracy of models.

\section{A. Lagrangian statistics}

Turbulent fluctuations induce extremely different Lagrangian behaviors of evaporating droplets. Figure 11 reports the histories of droplet positions, square radius, $r_{d}^{2} / r_{d, 0}^{2}$, and saturation, $S_{d}$, for two droplets showing opposite dynamics. The former, droplet 1, evaporates in about the half time of droplet 2. Both are initially nested into a fully saturated region located at inlet. Nevertheless, droplet 1 quickly moves into the dry environment surrounding the jet core $(r / R>1$ near the inlet), thus sampling a lower saturation level, $S_{d} \simeq 0.5$. Conversely droplet 2 remains entrapped into the turbulent jet core for a long time experiencing a high level of saturation which is weakly lowered by the turbulent mixing. Hence droplet 2 reaches a distance of about three times that of droplet 1 before fully evaporating. In order to statistically characterize the droplet vaporization dynamics, we consider the probability density function of the vaporization length and time computed over the whole droplet population. In analogy with the overall vaporization length previously introduced [Fig. 5(a)], the single-droplet vaporization length will be defined as the axial distance from the jet inlet, $z_{e}$, necessary for the droplet radius to decrease from $r_{d, 0}$ to a threshold radius $r_{d, t h}=0.1 r_{d, 0}$ (99.9\% of mass evaporated). The vaporization time $t_{e}$ is the corresponding amount of time. The PDFs of the droplet evaporation length and time are reported in Figs. 12(a) and 12(b), respectively. The mean, standard deviation, skewness, and kurtosis are reported in Table III and show a nearly Gaussian behavior with significant standard deviations. We note that a Gaussian behavior in turbulent flows is usually associated to fluctuations induced by the large-scale motions. However, it is remarkable how different the droplet histories are: half of injected droplets are present at about $z / R \simeq 30$ where about $90 \%$ of the injected liquid mass fraction is evaporated [see Fig. 5(a)]. This aspect is connected to the high polydispersity developed by the droplets in their downstream evolution.

Figure 13 shows the joint probability density function (JPDF) of the droplet vaporization lengths and times computed over the whole droplet population. A strong linear correlation is observed 


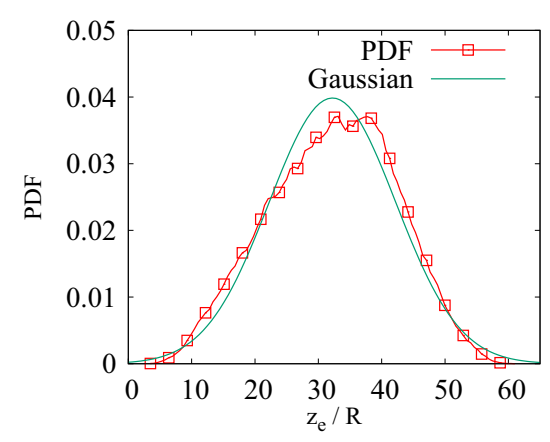

(a)

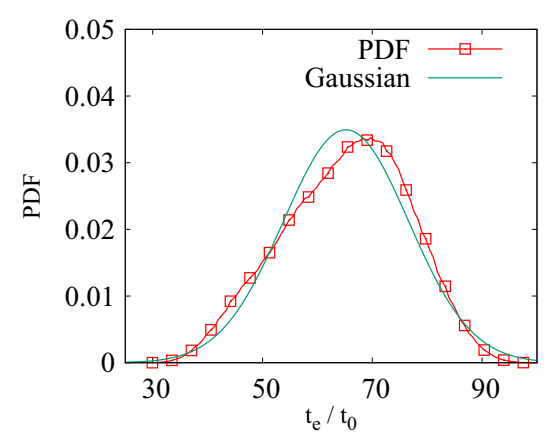

(b)

FIG. 12. (a) Probability density function of the nondimensional droplet vaporization length, $z_{e} / R$, with $R$ the jet inlet radius. (b) Probability density function of the nondimensional droplet vaporization time, $t_{e} / t_{0}$, with $t_{0}$ the reference time scale, $t_{0}=R / U_{0}$. The PDFs are computed over the entire droplet population injected into the computational domain. See the text for detailed definition.

between the droplet vaporization length, $z_{e} / R$, and time, $t_{e} / t_{0}$. We note that there is a certain number of droplets that completely evaporate in the spray zone located between the jet inlet and $z / R \simeq 10$, but survive in the liquid state for a considerable amount of time before being completely vaporized, $t_{e} \geqslant 30 t_{0}$. This behavior can be explained considering that droplets injected close to the wall at the nozzle edge have low axial velocities. In particular, these droplets originate from the viscous sublayer of the inlet turbulent pipe and move away from the saturated spray core towards the outer dry environment due to the radial velocity fluctuations induced by the turbulent carrier phase. Figure 14 shows the JPDF of the droplet axial velocity and radial position conditioned on the droplets which quickly evaporate, i.e., with $z_{e} \leqslant 10 R$. The largest part of these droplets slowly moves in the flow direction with axial velocities in the range $0<u_{d, z}<0.2 U_{0}$. This velocity range is comparable to the mean velocity of the viscous sublayer in the injection pipe, $U_{v s} / U_{0}=2.5 u_{\tau} / U_{0} \simeq 0.17$, with $u_{\tau}=0.067 U_{0}$ the friction velocity in the pipe and $U_{0}$ the bulk velocity. At the inflow section these droplets are surrounded by a fully saturated gas that prevents the vaporization process from starting. However, as they move away from the fully saturated jet core towards the outer dry environment their vaporization rate increases. The evaporation time of this subset of droplets can be estimated through the d-square law:

$$
\frac{t_{e}}{t_{0}}=\frac{\operatorname{ReSc}}{\operatorname{Sh}} \frac{\rho_{l}}{\rho_{g}} \frac{r_{d, 0}^{2}}{R^{2}} \frac{1}{H_{m}}, \quad H_{m}=\ln \left(\frac{1-Y_{v}}{1-Y_{v, s}}\right) .
$$

The physical parameters reported in Table I and the average value of $H_{m} \sim 0.052$ (computed on the droplets that completely evaporate with $z_{e} / R \leqslant 10$ ) lead to an estimated vaporization time $t_{e} \simeq 30 t_{0}$, which is consistent with present results. It emerges that even droplets which evaporate at short distances from the inlet need a relatively long time to escape from their initially nearly saturated conditions. Figure 15(a) shows the probability density function of the droplet radius at different axial

TABLE III. The mean, $\mu$, standard deviation, $\sigma$, kurtosis, $K$, and skewness, Sk, of the PDFs of droplet evaporation length and time with $\mu=E[X], \sigma=\sqrt{E\left[(X-\mu)^{2}\right]}, K=E\left[(X-\mu)^{4}\right] / E\left[(X-\mu)^{2}\right]^{2}$, and Sk $=$ $E\left[(X-\mu)^{3}\right] / E\left[(X-\mu)^{2}\right]^{3 / 2}$. All variables are nondimensional.

\begin{tabular}{ccccc}
\hline \hline & $\mu$ & $\sigma$ & $K$ & Sk \\
\hline$z_{e} / R$ & 30.64 & 10.22 & 2.39 & -0.010 \\
$t_{e} / t_{0}$ & 60.22 & 14.06 & 2.71 & -0.378 \\
\hline \hline
\end{tabular}




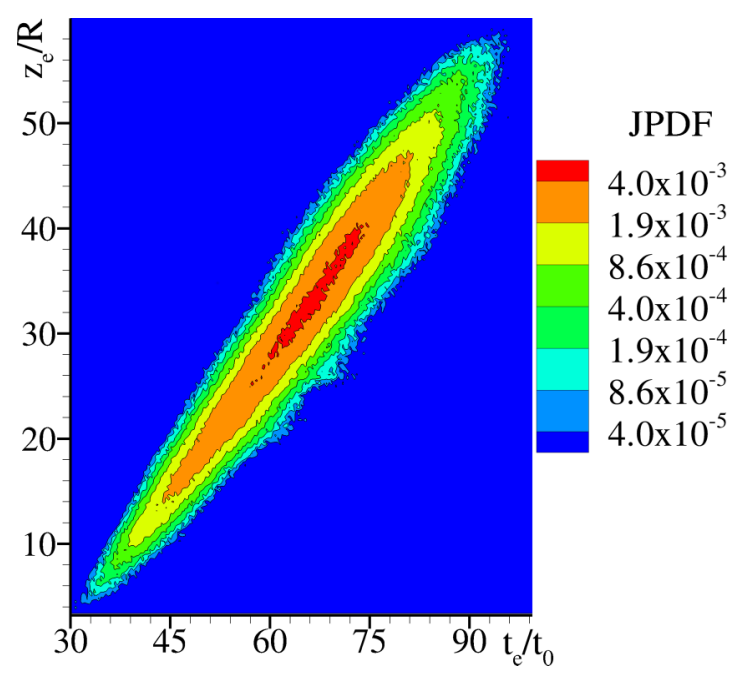

FIG. 13. JPDF of the vaporization lengths and times of the droplets computed over the whole droplet population. See the text for detailed definition.

distances from the origin. Even starting from a monodisperse suspension, we suddenly observe a radius distribution which spans around one decade after 10 jet radii from the inlet (the PDF sharply ends because droplets with radius $r_{d}<0.5 \mu \mathrm{m}$ are removed from the simulation). It should be remarked that this quantity results in differences of droplet volumes of about $10^{3}$. Wide droplet size distributions are consistent with experimental observations in turbulent sprays [32]. We attribute this intense spread of the droplet size spectrum to the strong fluctuations of the vapor concentration felt by the droplets because of the complex preferential segregation dynamics. This finding could be critical in modeling where polydispersity is often not considered. The heterogenous conditions shown by droplets which travel in an inhomogenous field and in aggregates with different sizes and histories are reflected in the wide variations exhibited by the vapor saturation level at the droplet surface; see Fig. 15(b). At $z / R=10$, the PDF shows values ranging between zero, i.e., dry gas, and above 1 , i.e., supersaturation with condensation. Further downstream, $z / R=20$, the spreading trend inverts

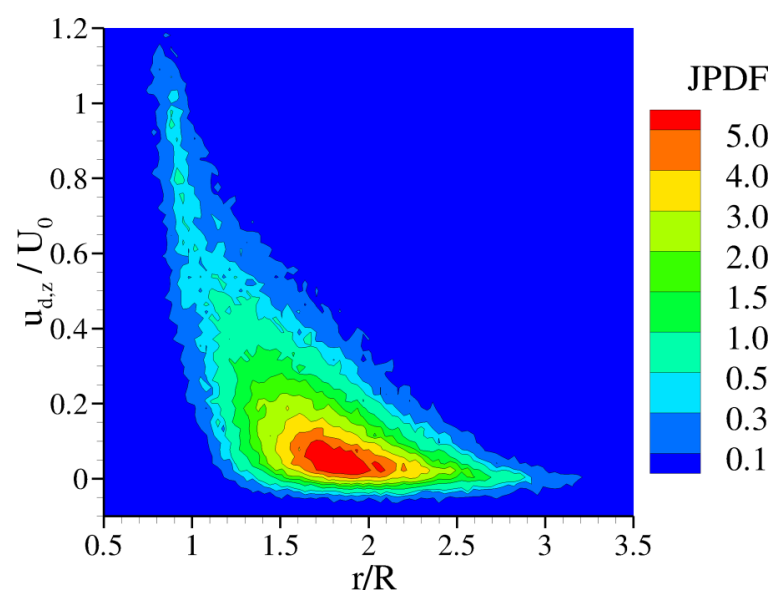

FIG. 14. JPDF of the droplet axial velocity, $u_{z} / U_{0}$, and radial position, $r / R$, computed over the droplets that have a vaporization length $z_{e} / R<10$. 


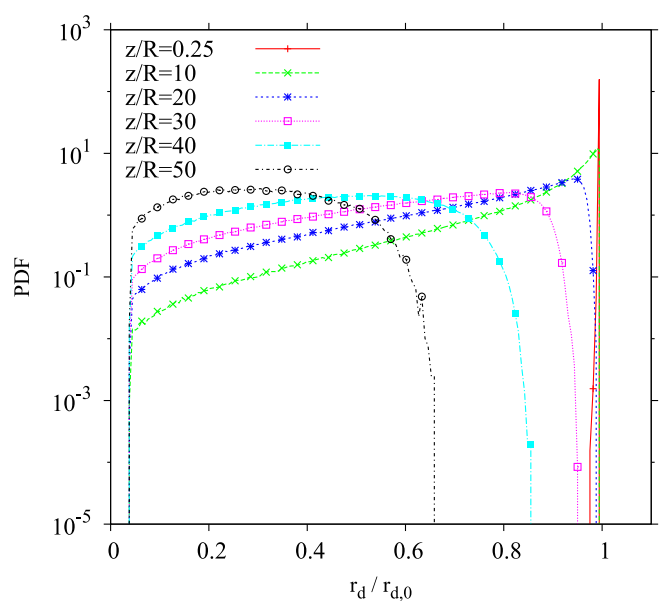

(a)

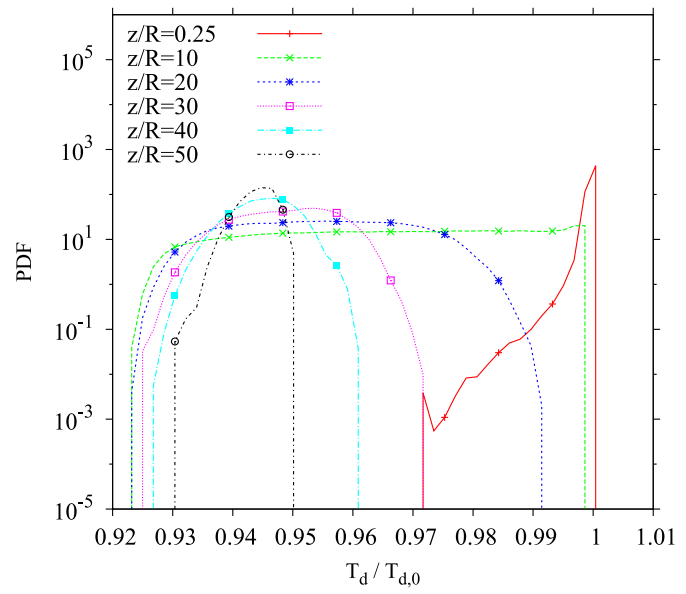

(c)

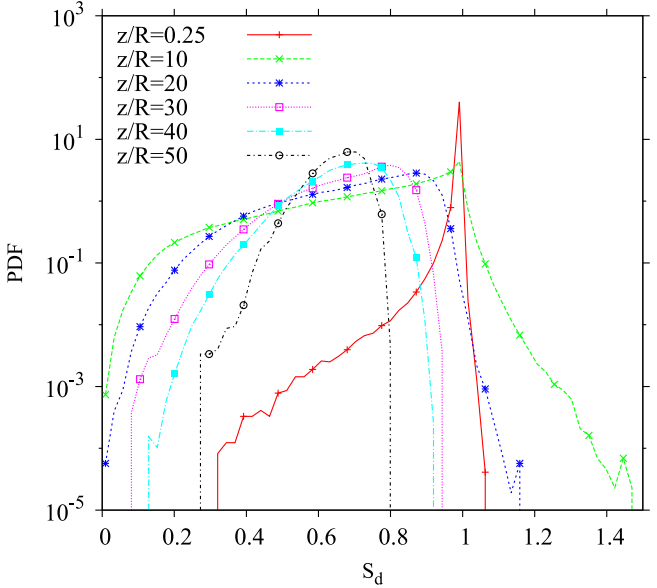

(b)

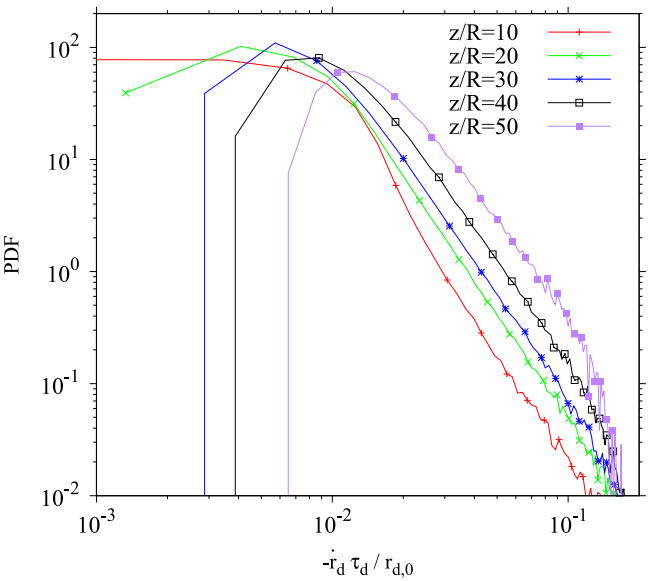

(d)

FIG. 15. PDF of Lagrangian observables. (a) PDF of nondimensional droplet radius, $r_{d} / r_{d, 0}$, where $r_{d, 0}$ is the initial radius of the injected droplets. (b) PDF of the saturation field at droplet surface, $S_{d}=Y_{v, s} / Y_{v}$, where $Y_{v, s}=Y_{v, s}\left(T_{d}, p\right)$ is the vapor mass fraction at saturation computed as a function of the droplet actual temperature and the carrier phase thermodynamics pressure, $p_{0} . Y_{v}$ is the actual vapor mass fraction in the carrier gaseous mixture evaluated at droplet position. (c) PDF of nondimensional droplet temperature, $T_{d} / T_{d, 0}$, where $T_{d, 0}$ is the initial temperature of the injected droplets. (d) PDF of nondimensional droplet vaporization rate, $-\dot{r_{d}} \tau_{d, 0} / r_{d, 0}$, where $\tau_{d, 0}$ and $r_{d, 0}$ are the initial relaxation time and radius of the injected droplets. The PDF plots (a), (b), and (c) are log-linear, and plot (d) is log-log.

and droplets are subjected to progressively more uniform saturation levels. The different evaporation dynamics caused by the saturation level felt by the droplets induces a similar statistical behavior for the temperature PDF, reported in Fig. 15(c). The temperature PDF appears flat even at $z=10 R$ with the highest variance shown in the downstream evolution. In addition to differential diffusion processes, the observed supersaturation can be attributed to inertial effects of the droplet dynamics. An evaporating droplet tends to increase the saturation level because of two contributions. On one hand it emits vapor increasing the surrounding vapor concentration, $Y_{v}$, and on the other hand it reduces the saturated vapor mass fraction, $Y_{v, s}$, because of the lower droplet (and gas) temperature. For fast evaporating droplets, it can happen that the droplet temperature strongly reduces inducing 


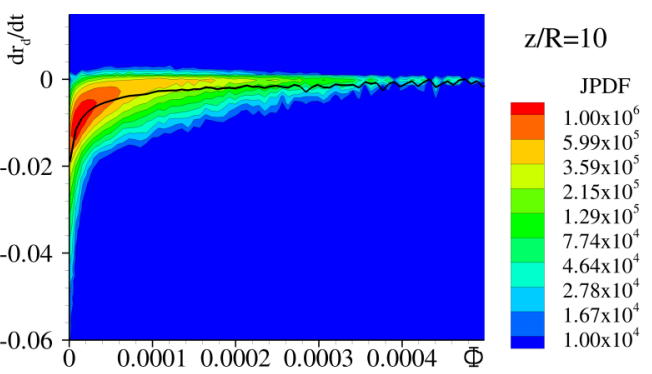

(a)

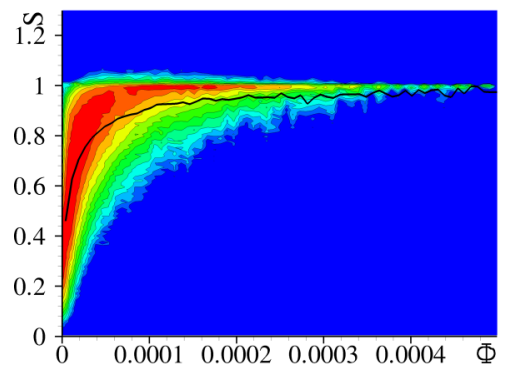

(c)

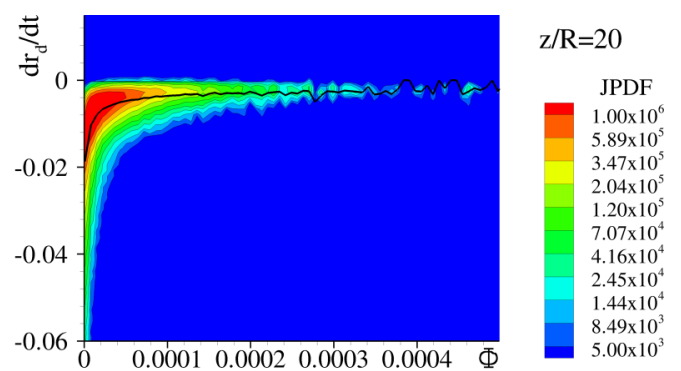

(b)

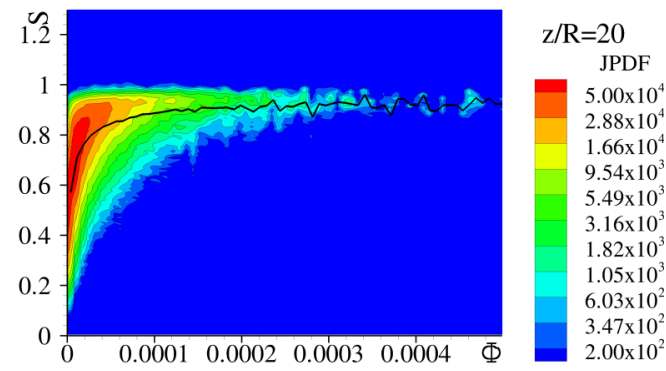

(d)

FIG. 16. Joint probability density functions of liquid phase volume fraction, $\Phi$, versus droplet vaporization rate, $d r_{d} / d t$ (a and b) and saturation field sampled by droplets, $S$ (c and d). Both statistics are computed at two different axial distances from the jet inflow, $z / R=10$ and $z / R=20$. Droplet vaporization rate is nondimensional. The reference scale is defined as $r_{d, 0} / \tau_{d, 0}$, with $r_{d, 0}$ and $\tau_{d, 0}$ initial droplet radius and relaxation time.

saturated conditions which block the evaporation even without high values of vapor concentration. Then, because of inertia, if the droplet moves into a higher vapor concentration region, still keeping its low temperature, supersaturation conditions occur. The PDF analysis shows how different are the histories of the initial monodisperse droplets in the turbulent spray. This strong heterogeneity should be accounted in models where few representative droplets are considered. The high polydispersity combined to the wide spectrum of saturation levels sampled by the droplets induces a nontrivial behavior of the vaporization rate PDF. Even though we cannot provide an argument, we find that the PDFs of the droplet radius change rate [Fig. 15(d)] appear to follow a power law with exponent about -3 , independent of the axial distance from the jet inlet and in turn on the local average conditions. This behavior means that extreme evaporating events are relatively frequent and are presumably excited by turbulent fluctuations. The liquid phase volume fraction around (sampled by) the droplets provides a measure of local preferential concentration of droplets themselves and can be contrasted to droplet vaporization rate, $d r_{d} / d t$, and saturation level sampled by droplets, $S$. The correlation among these quantities elucidates the effect of clustering on the local event of the evaporation dynamics. Figure 16 provides the joint probability density functions of these observables versus volume fraction sampled by droplets, $\Phi$. When droplets are not in clusters, i.e., low volume fraction $\Phi$, we note a clear correlation between vaporization rate $d r_{d} / d t$ with $\Phi$ and saturation level $S$ with $\Phi$. Nevertheless, at the same time we also observe a large variance of both vaporization rate and saturation level around their mean conditional values. This denotes that in dilute regions the vaporization rate is higher in average as expected, but large deviations from the mean are frequently induced by turbulent fluctuations of vapor concentration and temperature. We also note that, in dilute conditions, turbulent fluctuations may bring the local conditions at supersaturation levels, $S>1$, such that condensation occurs: $d r_{d} / d t>0$. Conversely, in regions where the liquid volume fraction is higher, the saturation sampled by droplets is distributed over a narrow range close to 


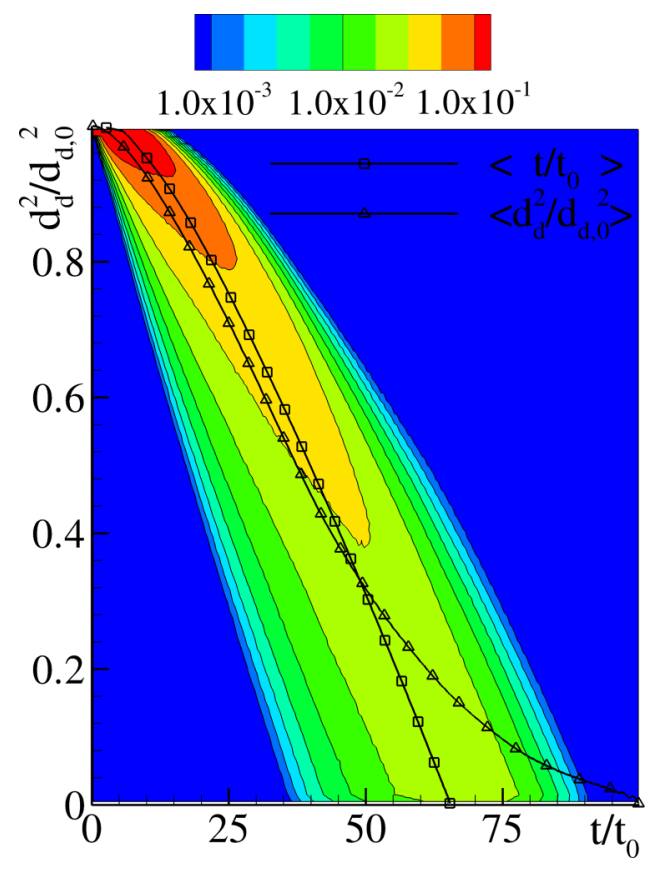

FIG. 17. Joint probability density function of droplet square diameter and flight time, $j f\left(t, d_{d}^{2}\right)$. Also the mean square droplet diameter as a function of the flight time, $\left\langle d_{d}^{2} / d_{d, 0}^{2}\right\rangle\left(t / t_{0}\right)$, and the mean droplet flight time as a function of the square droplet diameter, $\left\langle t / t_{0}\right\rangle\left(d_{d}^{2} / d_{d, 0}^{2}\right)$.

fully saturated conditions, $S=1$, with a nearly vanishing vaporization rate. This behavior can be attributed to a "collective inertia" induced by the high droplet concentration which tends to keep around nearly saturated conditions the local vapor phase. In these regions, indeed, a small perturbation of saturation is immediately balanced by an opposite fluctuation of overall evaporation rate due to the large number of droplets constituting the clusters. This mechanism rapidly brings the system to the local equilibrium. Hence, in these conditions, the evaporation dynamics shows small fluctuations around mean values and is always close to equilibrium.

The strongly inhomogenous conditions shown by the evaporating droplets impact on the possibility of a simple prediction of the spray vaporization length and time. In this context, the d-square law provides a valuable analytical tool often used to estimate the time evolution of the droplet size with the main assumption based on the reference environmental state, e.g., bulk vapor concentration. In these conditions, the d-square law estimates a linear behavior for the square of the droplet diameter, i.e., $\left\langle d_{d}^{2} / d_{d, 0}^{2}\right\rangle \simeq 1-k t / t_{0}$ with $k=\left(\rho \operatorname{Sh} R^{2}\right) /\left(\operatorname{Re} \rho_{l} \mathrm{~S} c r_{d, 0}^{2}\right)\left\langle\ln \left(1+B_{m}\right)\right\rangle$ the evaporation rate constant. Figure 17 provides the joint probability density function of the droplet radius and flight time, $j f\left(t, d_{d}^{2}\right)$. This latter parameter is computed as the time interval within the droplet injection time and the sampling one. The figure provides also the evolution of the average droplet square diameter versus time, $\left\langle d_{d}^{2} / d_{d, 0}^{2}\right\rangle\left(t / t_{0}\right)$, and the evolution of the droplet mean flight time as a function of the square droplet diameter, $\left\langle t / t_{0}\right\rangle\left(d_{d}^{2} / d_{d, 0}^{2}\right)$. It should be remarked that, by the definition of the JPDF, the mean square droplet diameter and mean flight time curves can be calculated as conditional averages:

$$
\begin{gathered}
\left\langle d_{d}^{2}\right\rangle(t)=\int_{0}^{\infty} d_{d}^{2} j f\left(t, d_{d}^{2}\right) d d_{d}^{2} \\
\langle t\rangle\left(d_{d}^{2}\right)=\int_{0}^{\infty} t j f\left(t, d_{d}^{2}\right) d t
\end{gathered}
$$


The mean square droplet diameter (black line with triangles) does not show any range where a linear behavior is attained. For small times, the deviation from the d-square law can be explained considering that droplets are injected in a saturated gas stream that needs some space (and time) before can be diluted via the jet entrainment process. Hence, the reference environmental conditions strongly change impacting on the assumption of the d-square law. For long times, the deviation from the linear profile can be understood considering the high polydispersity in combination with the prescribed positivity of the square droplet diameter. In this regime the most part of the droplets disappear because completely evaporated. These features induce the strong bending of the mean square droplet diameter from the linear behavior showing a heavy tail. Conversely, the mean flight time, $\left\langle t / t_{0}\right\rangle\left(d_{d}^{2} / d_{d, 0}^{2}\right)$, shows a closer agreement with a linear law if excluding the small time behavior. Although similar to the mean square droplet diameter up to $t / t_{0}<40$, then the flight time shows an approximatively linear trend from $t / t_{0}>30$. It should be noted that the mean flight time is not affected by the positivity of the square droplet diameter when a polydisperse distribution is considered. Hence, the mean droplet flight time appears as the most reliable observable in order to characterize and model the evaporation in jet sprays. However, we also note that using the d-square law with present environmental conditions for an a priori estimate of the vaporization time would give a value around $7 t_{0}$, which is smaller by one order of magnitude with respect to what has been found from DNS data. This large difference is the result of the complex heterogenous dynamics here described for the turbulent spray evaporation process.

\section{FINAL REMARKS}

A turbulent evaporating spray is investigated by means of a direct numerical simulation. The simulation reproduces an acetone-air spray evolving in an open environment considering dilute, nonreacting conditions and accounting for the full coupling between the two phases due to mass, momentum, and energy exchanges. The entrainment of external dry air is also accounted for. Liquid acetone monodisperse droplets are continuously injected within the turbulent gaseous phase at a bulk Reynolds number $\operatorname{Re}=U_{0} R / v=3000$. A complete description of both the instantaneous and average fields of Eulerian and Lagrangian observables is provided. The distribution of droplets is strongly inhomogeneous, and clustering is apparent. In particular, droplet clusters promote the formation of and persist in high vapor concentration regions reducing the vaporization rate. The intensity of the preferential segregation has been measured by the clustering index. Preferential segregation develops downstream the jet inlet, first in the mixing layer and then in the turbulent core. In particular, two different mechanisms driving the inhomogeneous droplet distribution are identified: inertial small-scale clustering and droplet segregation induced by the entrainment process across the turbulent-nonturbulent interface. The former one is the result of the competition between droplet inertia and the Stokes drag and is found to be responsible for droplets' preferential accumulation mainly in the spray core and in the far field evolution of the flow. The latter one mainly affects the mixing layer and consists in the entrapment of droplets in turbulent structures with high vapor concentration which are originated in the core and protract towards the droplet-free dry environment. Simultaneously, droplet-free dry air regions are engulfed in the jet core diluting it. This mechanism induces a high correlation between the vapor and droplet concentrations in the mixing layer. Both these processes affect the droplet dynamics and result in an oversampling of the vapor concentration experienced by each droplet, affecting also the overall vaporization length and time. Probability density functions of droplet observables have been reported at different axial distances from the inlet. An impressive increase of the droplet polydispersity is found to arise in the downstream evolution of the spray, resulting in an extreme widening of the droplets size spectrum. This intense spread is attributed to the heavy-tail PDF of the droplet radius vaporization rate which is the result of the complex dynamics coupling the droplet and the vapor concentration fields. Because of these strong inhomogeneities, the behavior of the mean square droplet diameter is found to be different from the classical d-square law. For long times, a nearly linear behavior is shown by the mean flight time, which appears the most reliable observable in order to characterize the droplet evaporation. 
The mechanisms discussed in the present study are expected to be important in all turbulent flows characterized by a mixing layer with entrainment of dry air, e.g., plumes and clouds. The proper modeling of these mechanisms is critical in order to improve LES and RANS model capabilities to accurately reproduce the turbulent vaporization dynamics for both reacting and nonreacting sprays.

\section{ACKNOWLEDGMENTS}

The authors would like to acknowledge the financial support through the University of Padova Grant No. PRAT2015 (CPDA154914), as well as the computer resources provided by the CINECA ISCRA C project: TaStE (HP10CCB69W) and the contribution of the COST Action Flowing Matter (MP1305).

[1] P. Jenny, D. Roekaerts, and N. Beishuizen, Modeling of turbulent dilute spray combustion, Prog. Energy Combust. Sci. 38, 846 (2012).

[2] J. Krüger, N. E. L. Haugen, D. Mitra, and T. Løvås, The effect of turbulent clustering on particle reactivity, Proc. Combust. Inst. 36, 2333 (2017).

[3] V. Raman and R. O. Fox, Modeling of fine-particle formation in turbulent flames, Annu. Rev. Fluid Mech. 48, 159 (2016).

[4] A. Attili, F. Bisetti, M. E. Mueller, and H. Pitsch, Formation, growth, and transport of soot in a threedimensional turbulent non-premixed jet flame, Combust. Flame 161, 1849 (2014).

[5] I. M. Kennedy, Models of soot formation and oxidation, Prog. Energy Combust. Sci. 23, 95 (1997).

[6] P. Marmottant and E. Villermaux, On spray formation, J. Fluid Mech. 498, 73 (2004).

[7] A. Ferrante and S. Elghobashi, On the physical mechanisms of two-way coupling in particle-laden isotropic turbulence, Phys. Fluids 15, 315 (2003).

[8] P. Gualtieri, F. Picano, G. Sardina, and C. M. Casciola, Exact regularized point particle method for multiphase flows in the two-way coupling regime, J. Fluid Mech. 773, 520 (2015).

[9] S. Elghobashi, On predicting particle-laden turbulent flows, Appl. Sci. Res. 52, 309 (1994).

[10] F. Toschi and E. Bodenschatz, Lagrangian properties of particles in turbulence, Annu. Rev. Fluid Mech. 41, 375 (2009).

[11] E. Calzavarini, M. Cencini, D. Lohse, and F. Toschi, Quantifying Turbulence-Induced Segregation of Inertial Particles, Phys. Rev. Lett. 101, 084504 (2008).

[12] J. Bec, L. Biferale, M. Cencini, A. Lanotte, S. Musacchio, and F. Toschi, Heavy Particle Concentration in Turbulence at Dissipative and Inertial Scales, Phys. Rev. Lett. 98, 084502 (2007).

[13] E. W. Saw, R. A. Shaw, S. Ayyalasomayajula, P. Y. Chuang, and Á. Gylfason, Inertial Clustering of Particles in High-Reynolds-Number Turbulence, Phys. Rev. Lett. 100, 214501 (2008).

[14] P. Gualtieri, F. Picano, and C. M. Casciola, Anisotropic clustering of inertial particles in homogeneous shear flow, J. Fluid Mech. 629, 25 (2009).

[15] J. Reveillon and F. X. Demoulin, Effects of the preferential segregation of droplets on evaporation and turbulent mixing, J. Fluid Mech. 583, 273 (2007).

[16] T. C. W. Lau and G. J. Nathan, The effect of stokes number on particle velocity and concentration distributions in a well-characterized, turbulent, co-flowing two-phase jet, J. Fluid Mech. 809, 72 (2016).

[17] F. Picano, G. Sardina, P. Gualtieri, and C. M. Casciola, Anomalous memory effects on transport of inertial particles in turbulent jets, Phys. Fluids 22, 051705 (2010).

[18] D. B. Spalding, The combustion of liquid fuels, in Symposium (International) on Combustion, Vol. 4 (Elsevier, Amsterdam, 1953), pp. 847-864.

[19] G. A. E. Godsave, Studies of the combustion of drops in a fuel spray-the burning of single drops of fuel, in Symposium (International) on Combustion, Vol. 4 (Elsevier, Amsterdam, 1953), pp. 818-830.

[20] M. R. Maxey and J. J. Riley, Equation of motion for a small rigid sphere in a nonuniform flow, Phys. Fluids 26, 883 (1983). 
[21] V. Armenio and V. Fiorotto, The importance of the forces acting on particles in turbulent flows, Phys. Fluids 13, 2437 (2001).

[22] S. Olivieri, F. Picano, G. Sardina, D. Ludicone, and L. Brandt, The effect of the basset history force on particle clustering in homogeneous and isotropic turbulence, Phys. Fluids 26, 041704 (2014).

[23] B. Abramzon and W. A. Sirignano, Droplet vaporization model for spray combustion calculations, Int. J. Heat Mass Transf. 32, 1605 (1989).

[24] C. Marchioli, M. V. Salvetti, and A. Soldati, Some issues concerning large-eddy simulation of inertial particle dispersion in turbulent bounded flows, Phys. Fluids 20, 040603 (2008).

[25] F. Mashayek, Direct numerical simulations of evaporating droplet dispersion in forced low Mach number turbulence, Int. J. Heat Mass Transf. 41, 2601 (1998).

[26] R. S. Miller and J. Bellan, Direct numerical simulation of a confined three-dimensional gas mixing layer with one evaporating hydrocarbon-droplet-laden stream, J. Fluid Mech. 384, 293 (1999).

[27] A. Bukhvostova, E. Russo, J. G. M. Kuerten, and B. J. Geurts, Comparison of DNS of compressible and incompressible turbulent droplet-laden heated channel flow with phase transition, Int. J. Multiphase Flow 63, 68 (2014).

[28] C. B. Da Silva, J. C. R. Hunt, I. Eames, and J. Westerweel, Interfacial layers between regions of different turbulence intensity, Annu. Rev. Fluid Mech. 46, 567 (2014).

[29] J. P. Mellado, Cloud-top entrainment in stratocumulus clouds, Annu. Rev. Fluid Mech. 49, 145 (2017).

[30] G. Falkovich, A. Fouxon, and M. G. Stepanov, Acceleration of rain initiation by cloud turbulence, Nature (London) 419, 151 (2002).

[31] G. Sardina, F. Picano, L. Brandt, and R. Caballero, Continuous Growth of Droplet Size Variance Due to Condensation in Turbulent Clouds, Phys. Rev. Lett. 115, 184501 (2015).

[32] Y. C. Chen, S. H. Stårner, and A. R. Masri, A detailed experimental investigation of well-defined, turbulent evaporating spray jets of acetone, Int. J. Multiphase Flow 32, 389 (2006).

[33] E. Villermaux, A. Moutte, M. Amielh, and P. Meunier, Fine structure of the vapor field in evaporating dense sprays, Phys. Rev. Fluids 2, 074501 (2017).

[34] A. Majda and J. Sethian, The derivation and numerical solution of the equations for zero Mach number combustion, Combust. Sci. Technol. 42, 185 (1985).

[35] J. G. M. Kuerten and A. W. Vreman, Effect of droplet interaction on droplet-laden turbulent channel flow, Phys. Fluids 27, 053304 (2015).

[36] F. Picano, F. Battista, G. Troiani, and C. M. Casciola, Dynamics of PIV seeding particles in turbulent premixed flames, Exp. Fluids 50, 75 (2011).

[37] G. Rocco, F. Battista, F. Picano, G. Troiani, and C. M. Casciola, Curvature effects in turbulent premixed flames of $\mathrm{H}_{2}$ /air: A DNS study with reduced chemistry, Flow Turbulence Combustion 94, 359 (2015).

[38] K. Fukagata and N. Kasagi, Highly energy-conservative finite difference method for the cylindrical coordinate system, J. Comput. Phys. 181, 478 (2002).

[39] J. G. M. Eggels, F. Unger, M. H. Weiss, J. Westerweel, R. J. Adrian, R. Friedrich, and F. T. M. Nieuwstadt, Fully developed turbulent pipe flow: A comparison between direct numerical simulation and experiment, J. Fluid Mech. 268, 175 (1994).

[40] G. Xu and R. Antonia, Effect of different initial conditions on a turbulent round free jet, Exp. Fluids 33, 677 (2002).

[41] G. D. Kinzer and R. Gunn, The evaporation, temperature and thermal relaxation-time of freely falling waterdrops, J. Meteorol. 8, 71 (1951).

[42] J. K. Eaton and J. R. Fessler, Preferential concentration of particles by turbulence, Int. J. Multiphase Flow 20, 169 (1994).

[43] J. Reveillon and F. X. Demoulin, Evaporating droplets in turbulent reacting flows, Proc. Combust. Inst. 31, 2319 (2007).

[44] R. A. Shaw, A. B. Kostinski, and M. L. Larsen, Towards quantifying droplet clustering in clouds, Q. J. R. Meteorol. Soc. 128, 1043 (2002).

[45] F. Battista, F. Picano, G. Troiani, and C. M. Casciola, Intermittent features of inertial particle distributions in turbulent premixed flames, Phys. Fluids 23, 123304 (2011). 